\title{
Coherent anti-Stokes Raman Scattering for Label-free Biomedical Imaging
}

\author{
Imran I. Patel ${ }^{\mathrm{a}, \mathrm{b}, \mathrm{ct}}$, Christian Steuwe ${ }^{\mathrm{a}, \mathrm{ct}}$, Stefanie Reichelt ${ }^{\mathrm{b}}$ and Sumeet \\ Mahajan $^{\mathrm{a}, \mathrm{c} *}$ \\ ${ }^{a}$ Cavendish Laboratory, University of Cambridge, Cambridge, CB3 OHE \\ ${ }^{\mathrm{b}}$ Cancer Research UK, Cambridge Research Institute, Cambridge, CB2 ORE \\ ${ }^{\mathrm{c} I n s t i t u t e}$ of Life Sciences, University of Southampton, Southampton, SO17 1BJ
}

\begin{abstract}
Coherent Anti-Stokes Raman Scattering (CARS) has established itself as an imaging technique capable of providing video rate imaging of biological specimens through vibrational coherence of endogenous molecules. Current techniques predominantly involve the application of costly, invasive and potentially non-specific dyes or labels for imaging biomolecules. CARS microscopy can however provide a high resolution and non-invasive alternative for imaging biomolecules of interest without the need for exogenous labels. Here we provide an overview of CARS including the technique and common instrumentation as well as its applications in biomedical imaging. We discuss the major biomedical areas where CARS has been applied such as in evaluating liver disease, progression of atherosclerosis, tumour classification and tracking drug delivery whilst also assessing the future challenges for clinical translation.
\end{abstract}




\subsection{Introduction}

For over 300 years optical microscopy has been one of the fundamental tools to study biology (1-3). In addition to the traditional bright-field visualization, many developments in microscopy have taken place over the years. Of particular note are phase contrast (4) and differential interference contrast (DIC) $(5,6)$ microscopy. While the former utilizes an optical mechanism (phase plate introduced in the focal plane of a microscope) to extract phase changes, the latter implements an interferometry configuration (separating and recombining beams using a Wollaston prism) to translate the differential response (optical path-length differences) into images. Both the techniques enable visualization of otherwise transparent objects on biological specimens without the need for stains or labels, however, they only give architectural information.

Staining (with dyes which can be fluorescent or non-fluorescent) has remained one of the main ways of molecular visualization and diagnostic imaging of cells ever since the first use of dyes in the midnineteenth century (7). The discovery of the green fluorescent protein (GFP) (8-10) and its variously coloured analogues (11) together with the evolution of molecular biology techniques which enabled their endogenous expression as labels in fusion constructs with proteins of interest changed the landscape of molecular imaging in cells. Much of the current understanding in cellular biology is owed to the remarkable success of these strategies coupled with fluorescence microscopy.

The foundation of fluorescence microscopy is the spectroscopy of the fluorochromes (fluorescent dyes, proteins and inorganic fluorophores) for visualising molecular processes in biology. Although fluorescence microscopy is widely used for detecting and imaging various biomolecules, it has one fundamental disadvantage: fluorochromes need to be attached or adsorbed on to them in order to be imaged (12). Therefore the success with fluorescence imaging relies heavily on the efficiency of the labelling or staining process. Expression of endogenous labelled proteins involves genetic modification of cells and arduous procedures sometimes involving a lot of trial and error. Moreover, whether the label modifies the functionality of the original molecule or affects other biochemical processes inside cells remains largely unknown. Fluorochromes used in staining are primarily used post-fixation although few live-cell stains are also available. Use of natural dyes is much preferred in staining solutions as some of the synthetic dyes can be hazardous ( 8 ) besides the fact that nonspecific binding is a significant issue with fluorescent stains especially for imaging small molecules such as drugs, metabolites and lipids (13). Additionally such small molecules although extremely important for a variety of biochemical processes in cells and tissues are difficult to be 
labelled without loss of function. Therefore a complimentary technique to fluorescence microscopy is highly desirable. Vibrational spectroscopy provides an alternative. Rather than using extraneously added fluorochromes, vibrational imaging generates contrast based on the intrinsic vibrations of molecules in their natural state inside the cell.

In vibrational imaging, molecular bonds with a certain characteristic resonance frequency are probed. Each molecule, due to its variety of distinct vibrational modes possesses a unique 'fingerprint' spectrum allowing its identification. Infrared (IR) absorption spectroscopy and Raman scattering are the most common techniques used to characterize chemicals and materials using their vibrational finger-print. While in IR spectroscopy micrometre radiation is used to directly excite molecular vibrations, in Raman spectroscopy, ultra-violet (UV), visible or near-IR radiation is used to observe scattering mediated by molecular vibrations. The inelastic interaction of a photon with a vibrational bond is referred to as Raman scattering (Fig. 1). Imaging with UV, visible or near-IR radiation results in better optical resolution than with IR. Moreover, crucially, IR spectroscopy is extremely sensitive to water which tends to obscure signals and limits penetration in biological specimens (14) unless samples are prepared carefully (dehydrated). However, water is a weak Raman scatterer and therefore Raman scattering is more suited to biomedical imaging.

Spontaneous Raman scattering can occur in two ways as shown in Figure 1: the transition can originate either from the vibrational ground state and generate a red shifted Stokes Raman photon (Fig. 1A) or from an excited vibration level leading to a blue shifted anti-Stokes Raman photon (Fig. 1B). Being a spontaneous process the intensity of the scattered radiation scales linearly with the power of the incident beam and the cross section of the process. The absolute Raman cross sections are typically in the range of $10^{-26}$ to $10^{-30} \mathrm{~cm}^{2}$ molecule ${ }^{-1}(15)$ thus around 6-10 orders of magnitude smaller than that of fluorescence. Thus Raman spectroscopy although powerful due to its inherent molecular characterisation and identification ability, non-invasive, non-destructive and label-free nature, is limited in applications especially in imaging due to its weakness.

Fortunately, weak Raman signals can be amplified by four wave mixing processes. In 1963 Minck et al. reported the generation of radiation at other frequencies due to a fourth-order process by focusing a Ruby laser in a cell filled with $\mathrm{H}_{2}$ atoms (16). It became clear that the lines of this radiation were dependent on vibrational-rotational transitions of the molecules. These processes are together referred to as nonlinear Raman scattering. The most prominent representatives are coherent antiStokes Raman scattering (CARS) and stimulated Raman scattering (SRS). In this review we focus on CARS and give an overview of its theory, technical and instrumentation aspects and detail the main areas in biomedicine to which CARS has been applied and is beginning to have an impact. In recent 
years there have been other reviews which cover CARS theory and its applications (17-19), which exemplify the exciting and expanding horizon of label-free bioimaging, to which the reader is also referred to for further details.

\subsection{CARS theory}

CARS bio-imaging was initially developed in 1982 where onion skin cells were studied by Duncan et al. (20) and saw a revival since the late nineties after developments in fast imaging sensitivity and solid state lasers (21-23). Due to the massive enhancement of the Raman cross section in CARS and SRS, acquisition times comparable to those of fluorescence microscopy could be achieved (24). These developments in sensitivity ultimately culminated in the demonstration of video-rate imaging with these techniques $(25,26)$.
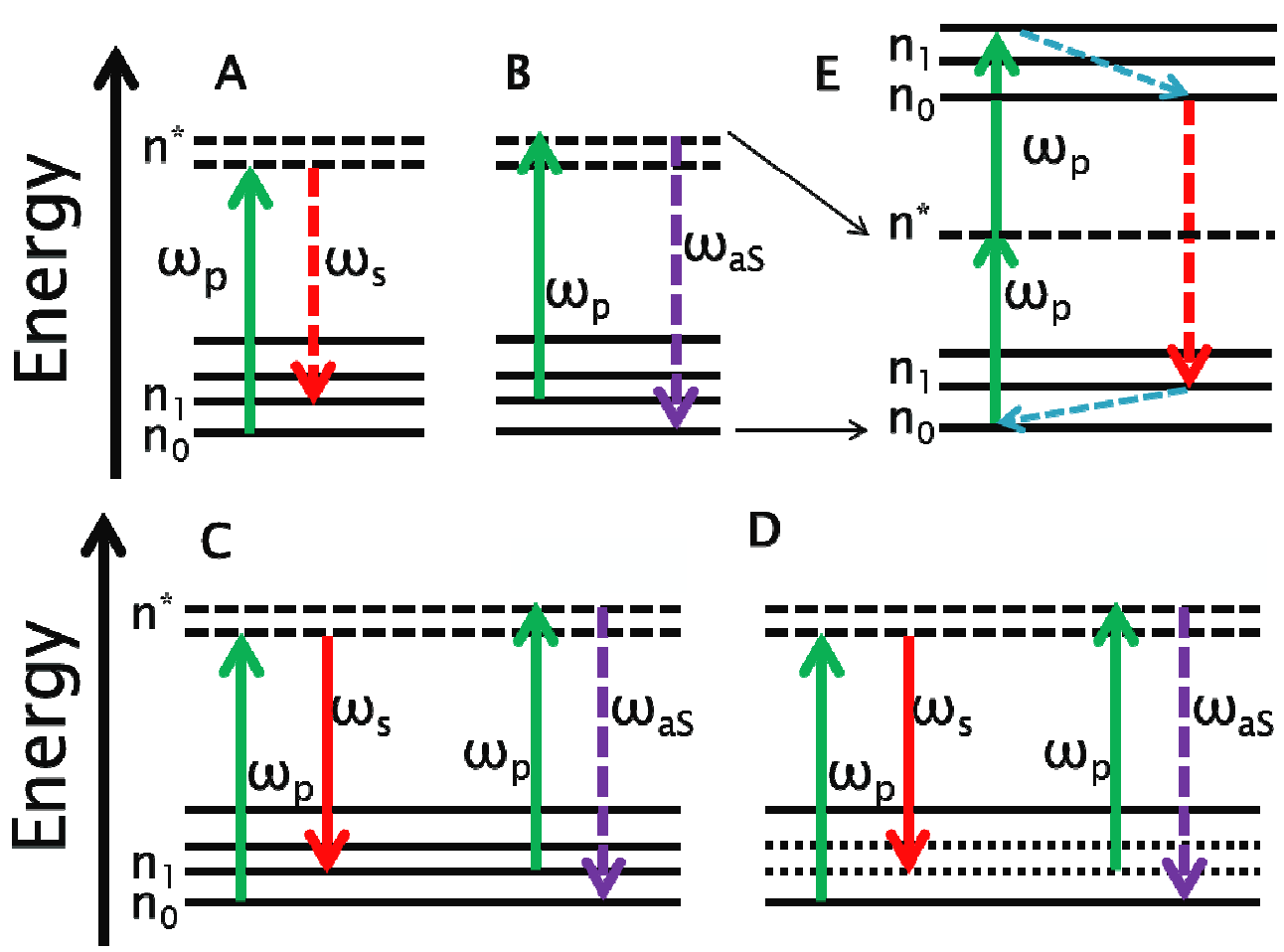

D

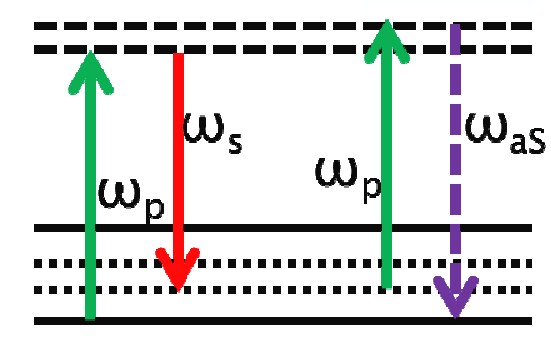

Figure 1. Schematic of Stokes, anti-Stokes Raman and CARS scattering at molecules. (A) During a Stokes Raman scattering process, a molecule is excited to a virtual level and relaxes to an excited vibrational level under emission of a Stokes photon $\omega_{s}$. (B) The anti-Stokes process starts from an excited state, hence the emitted photon $\omega_{\text {as }}$ is blue shifted. (C) Energy diagram for Coherent anti-Stokes Raman scattering (CARS). The CARS photon occurs at the anti-Stokes frequency $2 \omega_{p}-\omega_{s}$. All transitions happen on a short timescale of several 100 femtoseconds to picoseconds and via short-lived virtual states $n^{*}$. (D) Energy diagram for non-resonant CARS processes causing a background. The dashed lines indicate virtual states. (E) In two-photon fluorescence, each photon carries about half the energy required for exciting the fluorescent molecule. Typically, the emitted photon is shorter in wavelength than the two pump photons. 
In CARS, two pump photons and a Stokes photon interact simultaneously, mediated by a change in the third order polarisability, $\mathrm{P}\left(\chi^{(3)}\right)$, of the molecule generating an anti-Stokes photon $\left(\omega_{\mathrm{as}}\right)$ at $\omega_{\mathrm{as}}=$ $2 \omega_{p}-\omega_{s}$. Due to the stimulated nature of this effect, all molecular polarisations oscillate coherently, leading to a strong amplification of the signal compared to spontaneous Raman. Being a third order non-linear process, the CARS intensity is dependent on the cube of the pump and Stokes beams' initial intensities.

$I_{\text {CARS }} \propto N^{2} I_{\text {pump }}^{2} I_{\text {Stokes }}$

Due to its coherence, CARS is also proportional to the square of the Raman active molecules $N$ inside the focus. Therefore, high contrast is generated when CARS-active molecules are present inside cells in high local concentrations.

\subsection{Non resonant CARS Background}

A major drawback of CARS is the non-resonant background generated simultaneously. Although CARS conversion is very efficient if the difference frequency $\omega_{p}-\omega_{s}$ equals the vibrational resonance frequency (Raman shift) of a chemical bond, it also takes place under non-resonant conditions (Figure 1D). Instead of real vibrational Eigen states, electronic virtual states mediate the generation of a CARS photon. In general, the CARS third order susceptibility is expressed as a sum of a resonant frequency dependent part and a frequency independent non-resonant part (nr):

$$
\chi_{\text {CARS }}^{3}=\chi_{\text {res }}^{3}+\chi_{n r}^{3}
$$

In case the difference frequency $\omega_{p}-\omega_{s}$ does not match the frequency of a vibrational bond, the non-resonant contribution will dominate the CARS emission. The non-resonant part is present under all imaging conditions and therefore affects the sensitivity of the measurement.

\subsection{Other multi-photon effects}

The popularity of single photon fluorescence has accelerated the development of multi-photon fluorescence, where the energy of a single near infrared photon is not sufficient on its own to excite an electronic transition in the molecule. However, two simultaneously absorbed photons allow for this excitation to take place (figure 1E). Due to the strong non-linear origin of multi-photon processes, excitation is restricted to the femtolitre sized volume at the microscope focus. Ultimately this reduces photo bleaching and enhances the spatial resolution of all multi-photon processes including CARS 1): All multi photon microscopes exhibit inherent confocality. Unlike single photon fluorescence, multi-photon processes allow deep optical penetration such as investigations of 
disease models in living tissue due to excitation with long wavelengths in the near infrared regime (27). Another highly important non-linear process, also label-free, is second harmonic generation (SHG) generated in biological media giving insight into the extra cellular environment of tissues by detection of mostly collagen (28). Mediated by the second order polarisation, two photons incident at a non-centrosymmetric structure are converted into one photon with doubled energy, hence frequency. Both two-photon excitation fluorescence (TPEF) and SHG can easily be combined with current CARS setups since all techniques rely on similar laser technology. CARS, TPE and SHG emission often occur in well separated spectral regions and can easily be distinguished with appropriate filters.

\subsection{Technical and instrumentation aspects in CARS}

\subsection{Laser sources and imaging}

The strong non-linear nature of Raman processes in CARS requires sophisticated laser systems to provide two pulsed laser beams $\omega_{p}$ and $\omega_{s}$ (Fig. 1c) with high peak powers. Intuitively, shorter pulse durations give higher signals based on probability of higher order mixing processes. However, for CARS and SRS which rely on molecular vibrational transitions this is not the case. Typical Raman line widths $\left(\sim 10 \mathrm{~cm}^{-1}\right)$ are comparable to the spectral width of pulses with a couple of ps temporal duration. Numerical simulations show that the intensity based on the resonant part of the susceptibility saturates with shorter pulse durations (22). The non-resonant contribution however, has a quadratic dependence on the pulse duration. In the femtosecond regime, shorter pulses therefore generate a larger non-resonant contribution.

Many CARS $(22)$ and SRS $(29,30)$ setups hence do not use the whole spectral width of a $f s$ laser pulse for a pump or Stokes beam. For optimal spectral resolution ps pulses $\left(\sim 7 \mathrm{ps}, 3 \mathrm{~cm}^{-1}\right)$ have been recommended (31). Other groups are able to address long coherent times (ps range) of Raman resonances using $f_{s}$ pulses by shaping the spectrum of their temporal interference (32). This technique excites CARS processes efficiently by applying a linear chirp to both pulsed beams through glass dispersion.

Two main approaches for generating the two necessary laser beams have been developed: 1) Systems which utilize two separate lasers or a configuration of a laser and an optical parametric oscillator (OPO) and 2) Single laser and fibre based systems. 
3.1.1 CARS microscopes with two independently tuneable lasers

Titanium:Sapphire (Ti:Sa) lasers are tuneable over a wide range ( $650 \mathrm{~nm}-1050 \mathrm{~nm}$ ) and can be used in the picosecond configuration. The two lasers can be tuned into most Raman resonances from small shifts of several hundred wavenumbers up to the $\mathrm{CH}_{2}$ stretching region $\left(2800-3000 \mathrm{~cm}^{-1}\right)-\mathrm{a}$ huge advantage compared to other approaches (33). Both lasers have to be synchronised in time for a constant phase relation of the pulses (34). In a different approach, Mueller and Cheng et al. (35-37) and more recently Day et al. accomplished CARS microspectroscopy by using narrowband (10 ps, Spectra Physics Tsunami) and a broadband laser source (second Tsunami laser at $80 \mathrm{fs}$ ); this allowed acquisition of vibrational spectra over a $400 \mathrm{~cm}^{-1}$ bandwidth while scanning a sample (38).

\subsubsection{OPO based CARS microscopes}

Two lasers are not particularly cost effective and therefore most CARS setups use a single laser source which is split into two beams. One beam serves as the pump beam and the second is converted into either pump or Stokes beam by optical parametric conversion. OPOs are tunable over a wide range of wavelengths (39) and generate two beams (called the signal and idler) at lower frequencies such that their combined energy (of the 2 photons, respectively) equals that of the pump laser. A limitation of the Ti:Sa laser pumped OPOs $(21,22,40)$ is the gap between laser pump and signal wavelengths because of which Raman shifts below $2400 \mathrm{~cm}^{-1}$ are difficult to be imaged. Nevertheless, these high power OPOs are desirable for high speed video-rate imaging which keep pixel dwell times low. A lot of current setups use frequency doubled solid lasers at $532 \mathrm{~nm}$ pumping OPOs tuneable between $680 \mathrm{~nm}$ and $2150 \mathrm{~nm}$ (Levante Emerald, PicoEmerald, APE, Berlin) (25, 31, 41-43). In the latter case the output beam then serves as the tunable pump beam at a lower wavelength than the laser (Stokes) wavelength which is usually the fundamental laser wavelength of the solid state (e.g. Neodymium) laser at $1064 \mathrm{~nm}$. Freudiger et al. achieved powers of $<40 \mathrm{~mW}$ in both beams (31) which such a system, sufficient for fast CARS and SRS imaging. This OPO setup is shown in figure 2A. Current CARS systems may even have the laser, SHG unit and OPO built in the same system. Compact systems like the "picoEmerald" from APE Berlin deliver the two necessary beams and tune through almost all Raman shifts important for CARS imaging $\left(700-4500 \mathrm{~cm}^{-1}\right)$ in a fully automated manner. While these systems remain expensive, Zhang et al. showed that it is possible to successfully perform CARS imaging with an OPO built from scratch (40). 
A

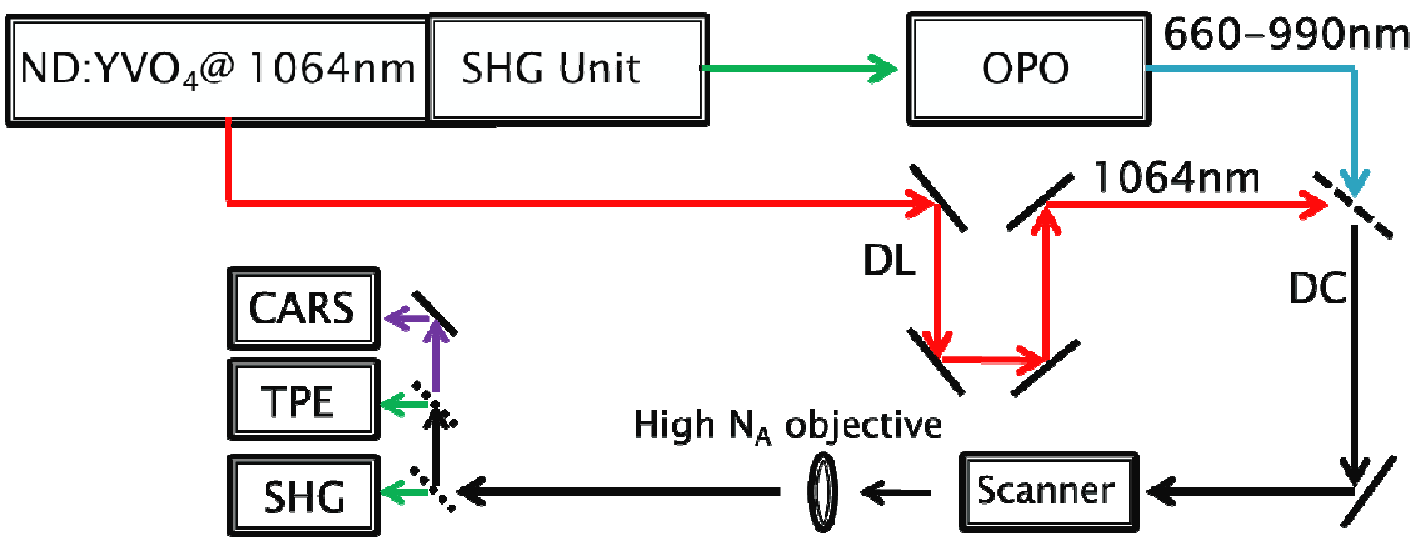

B

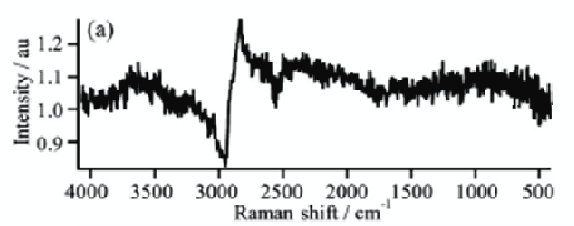

C
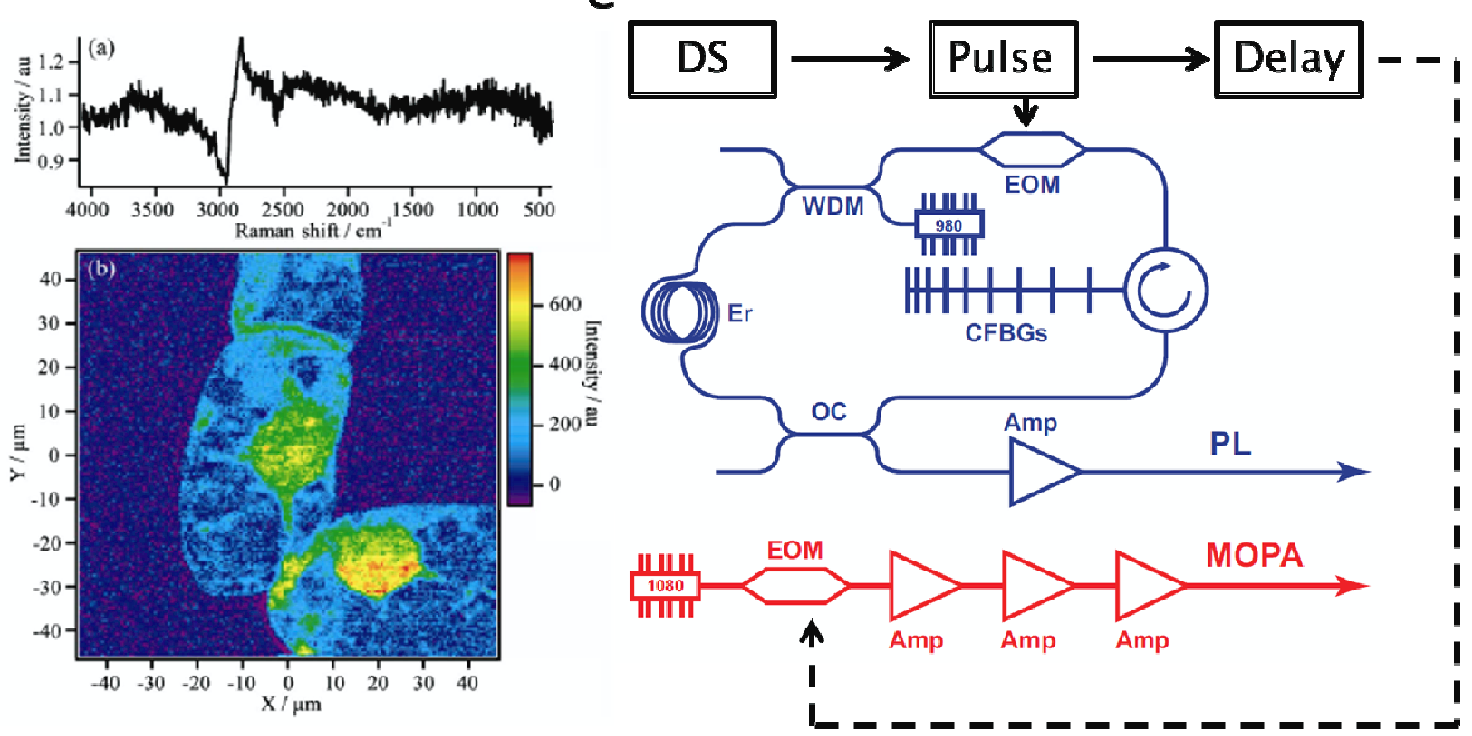

Figure 2. (A) Schematic of an OPO based laser system based on commercially available, tunable optical parametric oscillator (OPO) from APE pumped by a frequency doubled (SHG, second harmonic generation) $\mathrm{ND}: \mathrm{YVO}_{4}$ laser. The system allows multichannel detection of TPE, SHG and CARS signals. For CARS signal generation, (OPO) pump beam and the ND:YVO ${ }_{4}$ fundamental serving as Stokes have to be temporally overlaid by means of a delay stage (DL). (B) CARS micro-spectroscopy performed by Okuno et al. showing a CARS image at $2900 \mathrm{~cm}^{-1}$ (b) corresponding to a $\mathrm{C}-\mathrm{H}$ stretching bond taken by mapping the intensity difference between the peaks of the dispersive line shape in (a). This CARS spectrum corresponds to a position inside a nucleus. Image from ref. (44). Reproduced with permission. (C) State-of-the-art fiber based CARS laser system consisting of a programmable laser (PL) and a master oscillator (MOPA) serving as pump and Stokes sources. This system is based on high frequency, highly versatile function generator consisting of a digital synthesiser and a pulse generator driving electro optical modulators (EOMs). The delay of both pulses can be matched electronically. Frequency tuning is accomplished by varying the repetition frequency of pulses circulating in an Erbium fiber which changes the effective pulse dispersion in a chirped fiber Bragg grating (CFBG). Amplifiers (AMP) and diodes complete the setup. Image from ref. (45). Reproduced with permission. 


\subsubsection{Single laser and fibre based laser sources for CARS}

It is possible to use a single ultra-short pulsed laser ( $<10 \mathrm{fs}$ ) with a broad spectrum and pick Stokes and pump wavelengths individually by filtering or adding chirp $(32,46,47)$. In this latter approach the laser beam is split into lower and higher wavelength portions and care is taken to adjust the chirp of both pulses so that their instantaneous frequency difference remains constant. Changing the delay alters the instantaneous frequency difference and hence the Raman shift. Other approaches commonly implemented, due to their relative inexpensiveness compared to two laser/OPO systems, with fibres are described below.

\section{a) Super continuum based hyperspectral imaging}

Despite automated tuning, multicolour imaging by tuning OPOs is slow. Lim et al. report that their systems requires 20 minutes for a hyperspectral image stack to be generated (48). An easier approach towards multicolour and hyperspectral imaging is implemented by using instead of an OPO (in the scheme shown in Fig $2 \mathrm{~A}$ ) a continuum fibre (49-52) or even plain sapphire crystals $(29,30)$ to generate a continuum of Stokes wavelengths. With a typical continuum fibre, Stokes photons between $830 \mathrm{~nm}$ and $1100 \mathrm{~nm}$ are generated and thus can excite all possible CARS transitions of a molecule simultaneously. This broadband CARS approach allows taking a single spectrum within a few tens of milliseconds. An interesting hyperspectral CARS setup combined with a time resolved excitation scheme was published by Selm et al. By carefully adjusting the temporal delay of the excitation pulses and tailoring their shape, the non-resonant contribution of the CARS signal can be suppressed (53). Many designers of continuum fibre based systems claim that these fibres are perfect for applying them in nonlinear Raman imaging setups because of their wide spectrum (54). However, the overall power generated by continuum fiber is an integral over the whole spectrum. The power of a line of the spectrum is therefore small compared to that from an OPO and often too weak for fast imaging.

\section{b) Single line fibre based CARS systems}

A recent technological innovation for fast wavelength swept CARS hyperspectral imaging was introduced by Bégin et al. (45) A fibre based pump laser is combined with a fast, tunable programmable laser allowing up to 10000 flawless wavelength changes per second (45). In contrast to passive OPO systems, all parameters including pulse rate, wavelength, delay vs. the master oscillator are controlled by a high performance function generator which is programmed via 
software. A sketch of the laser design is shown in figure $2 \mathrm{C}$. The main limitation is the current available range of Raman shifts between 2700 and $2950 \mathrm{~cm}^{-1}$ only. However, the system is very flexible and can be used for imaging as well as generation of CARS spectra.

Pegoraro et al. developed a solution based on a single fibre based pump source at $820 \mathrm{~nm}$ and a highly non-linear fibre. One of the red shifted solitons generated inside the fibre was picked by a filter and its wavelength tuned by varying the input power (55). An all fibre based system has been shown by Baumgartl et al. who use a Ytterbium (Yb) tunable fibre laser generating 100 ps pulses which are converted to a wavelength pair at $780 \mathrm{~nm}$ and $1030 \mathrm{~nm}$ with $\sim 40 \mathrm{ps}$ pulse duration using a PCF (56). Unfortunately only CARS with Raman shifts around $3000 \mathrm{~cm}^{-1}$ is demonstrated but it should be possible to reach $2900 \mathrm{~cm}^{-1}$ after slight changes in their configuration. The same group demonstrates a second laser system which is a highly integrated all fibre system consisting of an $\mathrm{Yb}$ ps oscillator, however doesn't allow tuning through different Raman shifts. The fibre needs to be tailored for a specific pump frequency at a specified output (57). An interesting approach is presented by Gambetta et al. A femtosecond Erbium (Eb) based fibre oscillator is spectrally focussed and frequency doubled. A second portion is coupled into a super continuum generating fibre and its long wavelength peak compressed and doubled. Raman shifts from $300 \mathrm{~cm}^{-1}$ to $1000 \mathrm{~cm}^{-1}$ can be addressed. Wavelength tuning is accomplished by heating PPLN crystals (54).

\subsection{Suppressing the nonresonant background}

The non-resonant contribution increases quadratically with decreasing pulse width and hence, ps lasers sources are preferable for less non-resonant background generation. Further spectral pulse narrowing below the Raman line width of $10 \mathrm{~cm}^{-1}$ (24) however, decreases the signal for the same level of background signal. Epi-detection $(58,59)$ and the use of a collinear beam geometry $(21)$ have been shown to be beneficial. A popular background suppression technique relies on different polarisation properties of the resonant and nonresonant parts of the third order susceptibility (see equation 1). The nonresonant susceptibility is a real quantity in the system and independent of the Raman shift. The non-resonant CARS signal is linearly polarized. A polarizer which is placed perpendicular to that angle reduces the background very efficiently $(52,60)$.

\subsubsection{Heterodyne, interferometric and differential CARS}

Both Heterodyne and interferometric CARS distinguish between the resonant and non-resonant terms by mixing the CARS signal with a reference field from a local oscillator. The result is then 
dependent on the phase difference $\Phi$ between the CARS and the reference field, an interferometric mixing term. It is well known that the imaginary part of the CARS susceptibility contains the resonant information (61), hence is proportional to the signal in a spontaneous Raman spectrum. In heterodyne CARS, the phase difference $\Phi$ introduced by $\operatorname{Im}(\chi)$ is usually probed by phase modulating the reference field and evaluating the resulting amplitude modulation after interference of both beams although modulation of the pump beam itself can also be carried out, which results in twice the modulation of the detected CARS frequencies. A lock-in amplifier is then used to evaluate the detected signal and extract the resonant part (62-64). The implementation is slightly more involved

but the background suppression is efficient. In contrast to heterodyne CARS, in spectral interferometric CARS the necessary phase information is calculated from the spectral interference of a broadband reference CARS signal with the sample signal. Other variations of interferometry to remove the background have also been implemented such as in Fourier transform CARS (65).

In differential CARS, the same linear chirp to both pump and Stokes beams is added (32). This allows an effective instantaneous frequency difference (IFD) to be maintained between the chirped pump and Stokes beams. A different IFD can be addressed by tuning the delay between the beams. By generating two pulse pairs each exciting a different IFD, the resulting CARS intensities can be detected simultaneously by a single photomultiplier as sum and difference using phase-sensitive frequency filtering. The CARS intensities of both beams $I_{1}$ and $I_{2}$ are adjusted so that the differential CARS (D-CARS) signal is non-existent in the surrounding solution of a Raman active medium. Thus this also results in signals which are virtually background free (66).

\subsection{Detection}

\subsubsection{Epi and forward detection}

Unlike isotropic fluorescence, CARS and SRS radiation pattern depend on shape and size of the scattering object. According to Cheng et al. rod-shaped large objects, compared to the pump wavelength produce a cone of CARS radiation in forward direction whereas small, disk-sized objects generate both a forward and backward signal $(22,24)$. In the latter case, it is better to use epidetection since the non-resonant background from the surrounding medium radiates mainly in forward direction $(22,34)$. Most CARS setups have forward and backward detection installed and make use of multichannel detection to exploit all available information delivered by CARS. Hyperspectral CARS setups have to use a CCD detector in addition to a spectrograph in order to generate spectra. PMT sensitivities are better than those of CCD arrays at high scanning speeds of individual pixels (67). For ultrafast imaging, PMT based detection is vital to keep pixel dwell times low, alternatively, avalanche diodes (APDs) can be used. PMTs are useful for laser scanning owing to 
their large active area. Multicolour detection of CARS in combination with SHG and TPE can easily be implemented using appropriate dichroic long-pass and band-pass filters. Instantaneous sampling of modern data acquisition cards enables live observations using multiple photodetectors.

\section{CARS applications in biomedical imaging}

Within the biomedical field, spontaneous Raman spectroscopy has been extensively used to classify many diseases and identify potential markers of progression $(68,69)$, it has proven to be a highly valued tool for its label-free diagnostic potential (70) especially within the cancer field for its high accuracy classification of multiple types of tumour $(71,72)$. However, due to its low sensitivity, imaging requires long acquisition times per pixel in a raster scan fashion, these conditions are not ideal for use in biomedical applications such as live cell or in-vivo imaging. $(72,73)$. In contrast, CARS allows acquisition of 3D images down to depths of $400 \mu \mathrm{m}$ at video rate acquisition times, which is much more amenable to live cell imaging $(18,74,75)$. As described in the previous section extensive developments have taken place over the last decade in CARS technology improving its implementation and capabilities. CARS is increasingly recognized as a mature technique and therefore has rapidly growing applications in biomedicine. Moreover, the possibility of simultaneously implementing SHG and TPE on the same microscopy setup offers multimodality much suited to molecular imaging in cells and tissues. Some typical CARS systems and their applications in imaging different biochemicals are summarized in Table 1.

Table 1. Overview of commonly used CARS systems for imaging of biochemicals in cells and tissues

\begin{tabular}{|c|c|c|c|c|c|}
\hline $\begin{array}{l}\text { Excitation } \\
\text { source }\end{array}$ & OPO (nm) & $\begin{array}{l}\text { Pump and Stokes } \\
\text { wavelength (nm) }\end{array}$ & $\begin{array}{l}\text { Wavenumber } \\
\left(\mathrm{cm}^{-1}\right)\end{array}$ & Biochemical & $\begin{array}{l}\text { Biological } \\
\text { Application }\end{array}$ \\
\hline $\begin{array}{l}\text { 1064nm, 7ps, } \\
\text { Nd:YVO4 laser } \\
\text { (High-Q Laser) }\end{array}$ & $\begin{array}{l}680-980 \\
\text { (APE) }\end{array}$ & 817 and 1064 & 2845 & $\begin{array}{l}\text { Lipid } \\
\left(\mathrm{CH}_{2}\right)\end{array}$ & Cancer $(76,77)$ \\
\hline $\begin{array}{l}\text { 1064nm, 7ps, } \\
\text { Nd:YVO4 laser } \\
\text { (High-Q Laser) }\end{array}$ & $\begin{array}{l}680-980 \\
\text { (APE) }\end{array}$ & 953 and 1064 & 1095 & $\begin{array}{l}\text { DNA } \\
\left(\mathrm{PO}_{2}\right)\end{array}$ & $\begin{array}{l}\text { Nuclei } \\
\text { Morphology } \\
(78,79)\end{array}$ \\
\hline $\begin{array}{l}\text { 1064nm, 7ps, } \\
\text { Nd:YVO4 laser } \\
\text { (High-Q Laser) }\end{array}$ & & 817 and 1064 & 2845 & $\begin{array}{l}\text { Lipid } \\
\left(\mathrm{CH}_{2}\right)\end{array}$ & $\begin{array}{l}\text { Atherosclerosis } \\
(80) \text {; Lipid } \\
\text { metabolism in } \\
\text { worms (C. }\end{array}$ \\
\hline
\end{tabular}




\begin{tabular}{|l|l|l|l|l|l|}
\hline & & & & & elegans)(81) \\
\hline $\begin{array}{l}\text { 1064nm, 6ps, } \\
\text { Nd:YVO4 laser } \\
\text { (High-Q Laser) }\end{array}$ & $\begin{array}{l}680-980 \\
\text { (APE) }\end{array}$ & 811 and 1064 & 2930 & Protein & $\begin{array}{l}\text { Cell Apoptosis } \\
(82) \\
\left(\mathrm{CH}_{2}\right)\end{array}$ \\
\hline $\begin{array}{l}700-1000 \mathrm{~nm}, \\
\text { 100fs, Ti:Sa } \\
\text { laser (Mira 900, } \\
\text { Coherent) }\end{array}$ & $\begin{array}{l}700-1600 \\
\text { (APE) }\end{array}$ & 835 and 1095 & 2845 & Lipid & $\begin{array}{l}\text { Liver Steatosis } \\
(83)\end{array}$ \\
\hline
\end{tabular}

As CARS sensitivity depends on the square root of the concentration of the vibrational modes, a concentration of $10^{5}$ to $10^{6}$ vibrational oscillators in the focal volume is required to provide adequate contrast for imaging (84), thus biomolecules with the strongest Raman signals and large local concentrations are most suited to imaging. Lipid biomolecules are highly abundant in C-H chemical bonds and therefore provide high image contrast in living organisms; it is for this reason that they are the most widely studied for label free imaging using high resolution CARS microscopy $(17,85)$. Some examples of imaging liver and skin using multimodal non-linear imaging are shown in Figure 3. CARS in combination with other NLO imaging modalities has been utilised recently in many biomedical studies evaluating steatosis and fibrosis in liver $(83,86)$, progression of atherosclerosis in cardiac disease $(80,87)$, demyelination of the central nervous system (CNS) $(88,89)$, cancer classification $(77,90)$ and assessing the pharmacodynamics, delivery and efficiency of therapeutic drugs $(91,92)$. In subsequent sections we describe CARS studies in various biomedical areas. 

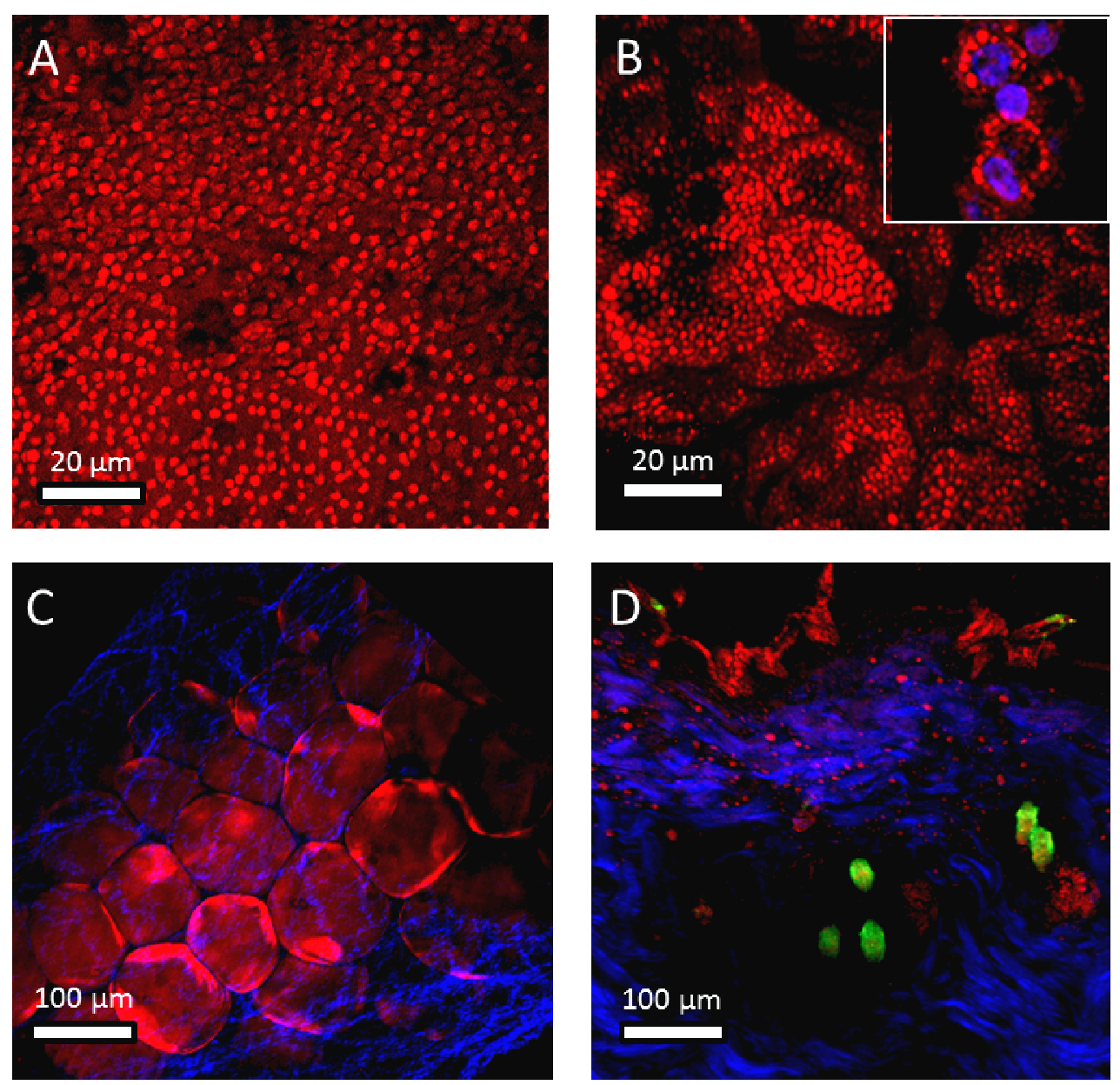

Figure 3. Non-linear optical imaging of biological tissues (z-stacks). (A) CARS imaging of lipid rich liver tissue. (B) CARS (red) and TPEF (blue) imaging of skin sebaceous glands illustrating sebum rich compartments within cells. The right hand corner image confirms presence of nuclei in cell areas devoid of lipids through Hoechst 33342 staining. (C) CARS (red) and SHG (blue) imaging of pancreatic adipocytes illustrating an outer collagenous matrix. (D) CARS (red), SHG (blue) and autofluorescence (green) imaging of skin tissue illustrating sebocytes/epidermis, collagen and hair shafts, respectively. These images from our lab have been acquired with an OPO based CARS system described in (93).

\subsection{In-vivo imaging of skin}

The skin is the largest organ in the body acting primarily as a protective barrier against chemical, physical and pathological insults. It can be divided into three main layers of epidermis, dermis and subcutis, interspersed with hair follicles, sebaceous glands and sweat glands (94). The epidermis is the avascular, outermost layer of the skin comprising several epithelial-cell types including keratinocytes (>90 \% cells), melanocytes, Langerhans cells and Merkel cells (mechanoreceptors). It can be subdivided into the following layers: stratum corneum, stratum granulosum, stratum 
spinosum and stratum basale averaging 60-70 $\mu \mathrm{m}$ in thickness in normal healthy skin $(95,96)$. Disorders, conditions and diseases of the skin can vary tremendously from alopecia, acne and psoriasis to malignant cancers, many of which commonly require the application of topical ointments to alleviate or treat the symptoms.

As the outer most surface of the body, skin is the most ideal tissue for live in vivo analysis. By demonstrating the strong epi-CARS signal backscattered from tissue, Evans et al. paved the way for CARS imaging by illustrating video-rate in vivo imaging of lipid $\left(\mathrm{CH}_{2}\right)$ rich structures such as adipocytes, sebaceous glands and corneocytes within the skin (25). By applying oil to the skin, the potential to track chemicals and their penetration was also shown to be feasible. In one of the first studies on clinical samples, CARS imaging visualised the distribution of topical ointment of omega-3oil over a specific time period by comparison of CARS images at $2845 \mathrm{~cm}^{-1}$ (lipids) and $3295 \mathrm{~cm}^{-1}$ (water). It illustrated that the oil entered through microscopic skin folds and accumulated at skin fold boundaries (97). In another study, Zimmerley et al. determined that $d$-glycine and water distributes homogenously through cortical regions of the follicular hair (98). Whilst more recently, imaging of healthy skin in comparison to psoriatic skin found differential lipid distributions at the stratum corneum making CARS imaging a valuable tool for providing insight into disease mechanisms (99). CARS imaging with regards to the skin has gained great interest as an in vivo approach to track the penetration of topical ointments and drugs. It is for this reason that CARS imaging would appeal widely to the pharmaceutical industry to assess the pharmacokinetics and penetration capabilities of cosmetic skin care products, ointments or drug over long term studies in vivo (100).

\subsection{Assessing atherosclerosis}

Atherosclerosis is a complex and progressive disease which involves the accumulation of lipids and cholesterol within the arterial walls. This multi-stage process involves an inflammatory response in which macrophages extravasate into the arterial walls and phagocytose low density lipoproteins (LDLs). The LDLs are unable to be broken down by the macrophages leading to gathering of phenotypical foam cells. Accumulation of LDL ultimately leads to foam cell rupturing and further increased macrophage infiltration, causing continuous progression of disease (101). As lipids are vital components of the atherogenesis process its morphology, composition and quantitation in plaque formation are fundamental to gaining new insights into cardiac disease $(102,103)$. Current clinical diagnostic techniques involve non-invasive methods such as computerised tomography and magnetic resonance imaging which require contrast agents and have low resolution hindering the capability to detect early stages of atherogenesis (104). 
CARS imaging is capable of providing mechanistic information in a label-free manner at a subcellular, cellular and tissue level without the requirement of contrast agents. Several studies have therefore employed multiplex CARS and associated multiphoton imaging techniques for the further elucidation of atherosclerotic plaque formation. Ko et al. illustrated the application of CARS to image atherosclerotic plaques (Fig. 4) in myocardial infarction prone rabbits (105). In a diet based study, Lim et al. characterised the effect of varied fat intake on early stage II/III plaque formation in ApoE-/mice using SHG to determine collagen content, TPEF to determine elastin fibers and CARS imaging for lipids (106). Westernised diets (high fat, high cholesterol) illustrated a 2 fold increase in arterial plaque area in comparison to a low fat standard diet. High magnification CARS images also illustrated clearly defined macrophages with distinct nuclei and lipid rich cytoplasm, additionally verified by CD68 macrophage staining. Interestingly, SHG identified a 4-fold decrease in collagen distribution in western diet plaques, potentially caused by macrophage induction and matrix metalloproteinases $(106,107)$. Further studies using hyperspectral CARS imaging not only identified lipid rich macrophages but also allowed distinction of lipid structure types such as needle and platelet shaped cholesterol crystals (48). Cholesterol crystals in plaques were evident with a significantly greater contribution in wild-type than in ApoE-/- knockout mice, suggesting a potential role of ApoE-/- in cholesterol crystal inhibition. Through compilation of the previous CARS based evidence, Kim et al. quantified 4 representative types of atherosclerotic lipids of needle and platelet shaped cholesterol crystals, extracellular lipid deposits and lipid foam cells in different stages of atherosclerosis (80). Lipid foam cells and extracellular lipids were present throughout each stage with significantly increased amounts of lipids at each stage; needle/platelet shaped cholesterol crystals were only present in advanced stages in the lesser curvature of aortic arch and innominate artery. Using CARS Duewell et al. implicated cholesterol crystals to have an effect on the plaque rupture susceptibility with its various forms and also presented evidence indicating its potential to activate macrophages through NLRP3 inflammasome (102). 

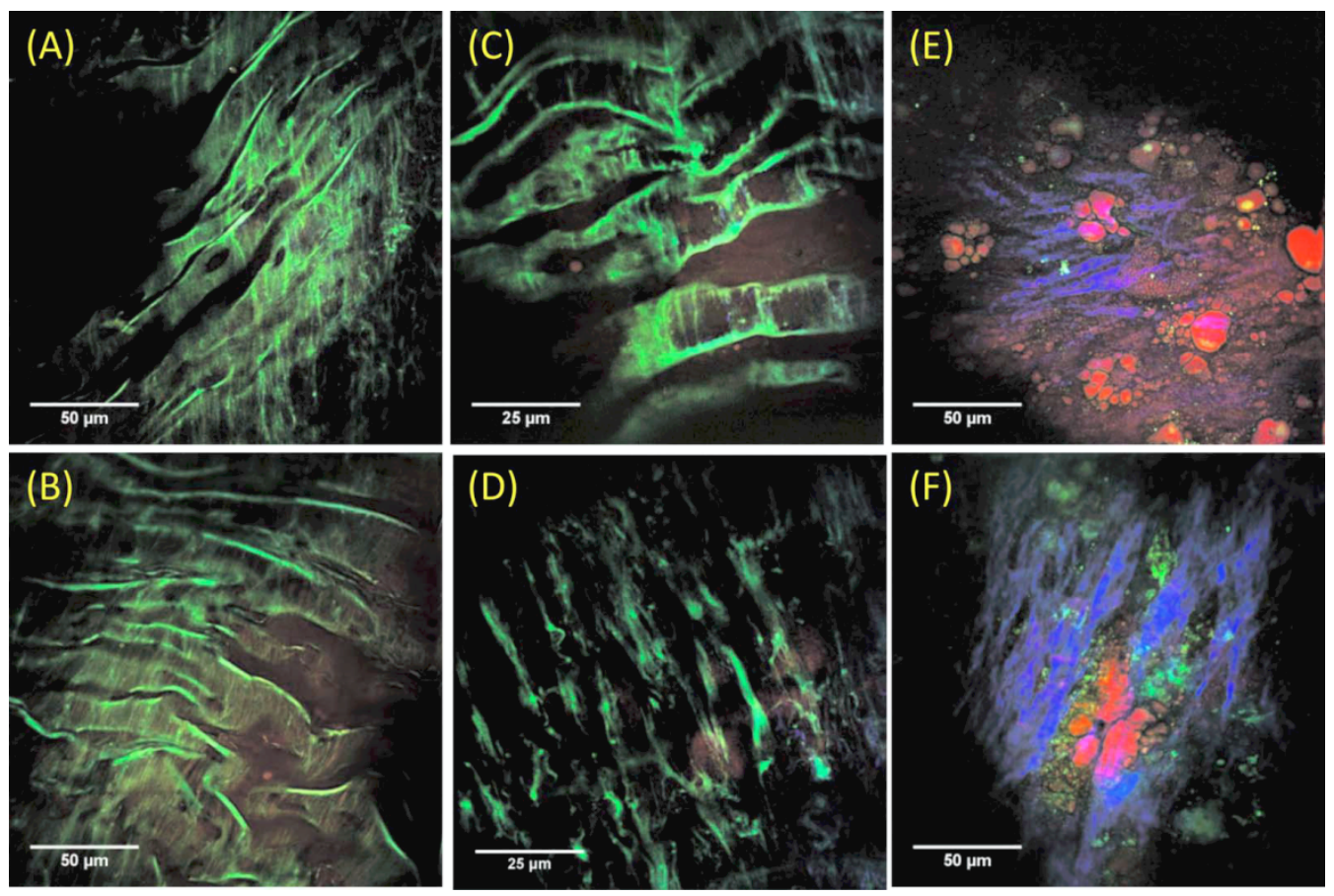

Figure 4. Representative epi-NLO images collected from healthy arterial lumen on A) 4-month-old and B) 10month-old atherosclerosis-induced myocardial infarction prone Watanabe heritable hyperlipidemic (WHHLMI) rabbit using 20x air objective lens, and on a 4-month-old rabbit using 40x WI lens at C) lumen surface and D) $\sim 20 \mu \mathrm{m}$ depth from surface. Representative epi-NLO images of arterial lumen surface obtained from E) an early atherosclerotic lesion and F) an advanced atherosclerotic lesion using 20x air lens. Green:TPEF elastin or other fluorescent particles. Blue:SHG collagen. Red:CARS lipid-rich structure. Image from reference (105). Published with permission.

\subsection{Diagnosis of liver disease}

The liver is a central organ for metabolic homeostasis whilst also playing a major role in detoxification. Biochemical pathways of gluconeogenesis, lipogenesis and cholesterol metabolism are constantly occurring making the liver a vital site for the synthesis, metabolism, storage and translocation of proteins, lipids and carbohydrates (108). Metabolic syndrome and obesity causes alteration in these metabolic pathways which can lead to pathophysiological changes within the liver known as non-alcoholic fatty liver disease (NAFLD) which can ultimately lead to hepatocellular carcinoma (109). Hepatic steatosis, abnormal accumulation of lipids, is an early stage sign for the diagnosis NAFLD or hepato-toxicity resulting from the accumulation of lipids in liver tissue. Current gold standard techniques to diagnose the severity of steatosis are carried out through histopathology from excised tissue sections (110). Inaccuracies in clinical diagnosis can occur through chemical processing of tissue using xylene which inevitably removes lipids from sections, 
whilst use of the ORO stain can also result in overestimation of steatosis as this is not specific to lipid droplets $(111,112)$.

Several studies have highlighted the use of CARS as a label free approach to provide improved accuracy and early diagnosis through taking advantage of its ability to image lipids without exogenous labels. Using a multimodal imaging system Lin et al. explored the fibrosis and steatosis effects of bile duct ligated rats (83). As a result, significant accumulation upto week 4 of lipid droplets was identified by CARS, whereas increased collagen fibrils upto week 6 was identified by SHG in association with severe hepatocyte necrosis. These findings demonstrate that there are potential differential rates of development for steatosis and fibrosis in NAFLD. More recently, Le et al. induced hepatotoxicity by feeding wild type and transgenic mice with fenofibrate (a common drug) to impair mitochondrial function and induce pathological onset of microvesicular steatosis. This study showed that CARS analysis on unstained sections had increased sensitivity to steatosis not only for severe cases but also for mild cases (see figure 5). On the contrary, ORO stained tissue sections were only sensitive to severe cases of steatosis (86). 

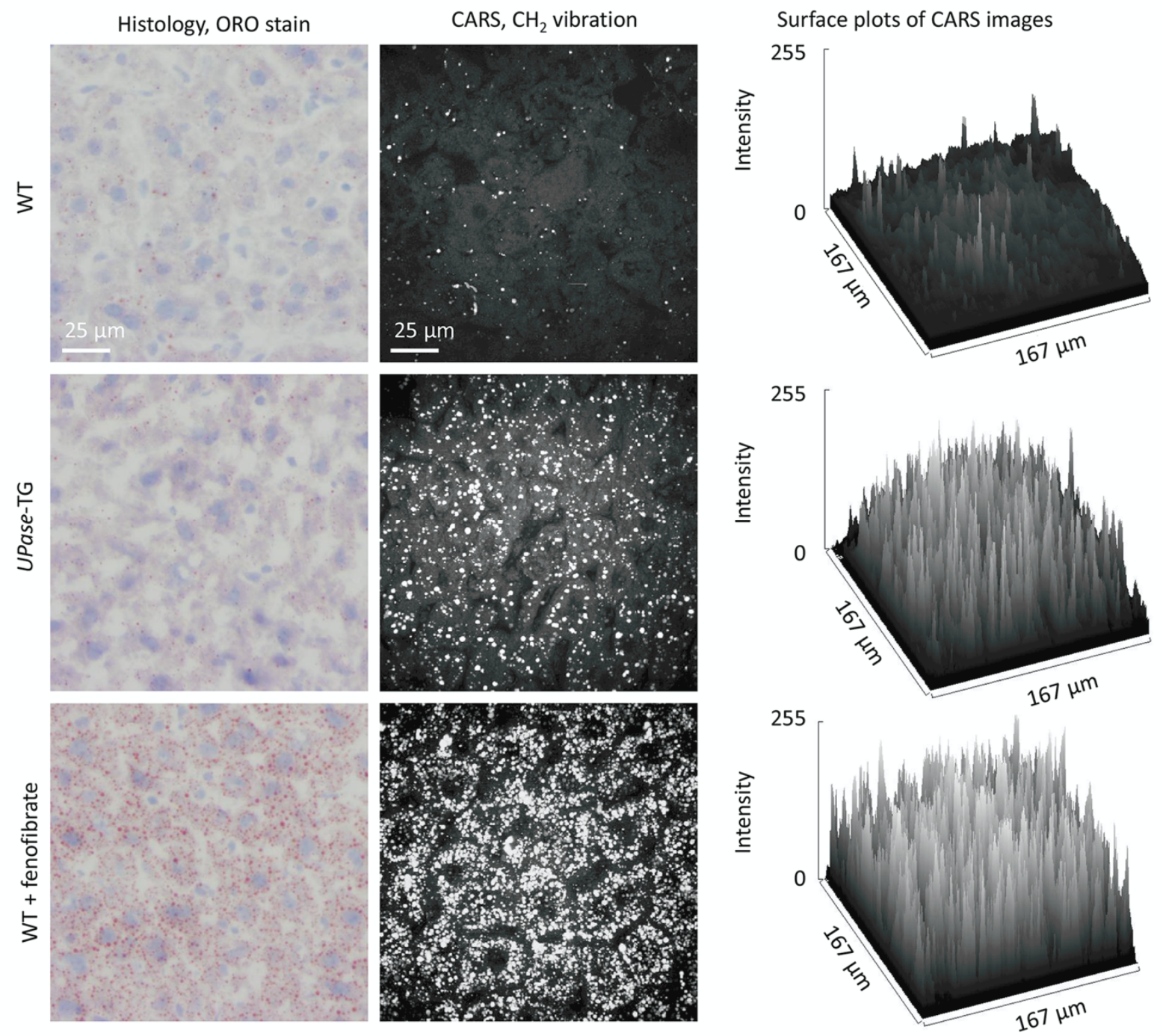

Figure 5. Detection of hepatic microvesicular steatosis with ORO histology and CARS microscopy. Representative ORO histology images (left column), CARS images (middle column), and surface plots of CARS images (right column) for wildtype mice with no steatosis (upper row), UPase-TG mice with mild microvesicular steatosis (middle row), and wildtype mice fed with $400 \mathrm{mg} / \mathrm{kg} /$ day fenofibrate for 5 days with severe microvesicular steatosis (lower row). Three mice, thus 3 livers, per animal group were used for direct comparison of ORO histology and CARS imaging analysis of microvesicular steatosis. Surface plots were performed with ImageJ software. Image from reference (86). Reproduced with permission.

\subsection{Classifying cancer and mechanisms of invasion}

\subsubsection{Cancer classification}

Turnaround times for cancer diagnosis and classification is pivotal towards the prognosis of the disease and the therapeutic treatment provided. Currently the gold standard clinical diagnostic test for cancer classification is surgical excision and conventional histopathological analysis, a procedure which has long turnaround times of a few days. If insufficient material has been retrieved or a 
tumour free margin has not been obtained, repeat surgery is required which is time consuming and expensive (113). Alternatively, a mis-diagnosis could lead to unnecessary or postponed treatment for the patient. Several studies have utilised CARS imaging for cancer diagnosis which can provide a rapid histological diagnosis $(76,77)$.

Through exploitation of CARS contrast for lipid distribution $\left(2845 \mathrm{~cm}^{-1}\right)$ in cells, morphological identification of the cell nuclei is also possible due to predominance of compact DNA instead of lipids in them; therefore they show a lower concentration of $\mathrm{C}-\mathrm{H}$ stretching intensities resulting in negative contrast in lipid distribution images. Yang et al. utilised this contrast to quantify morphological and geometrical information of cell nuclei using tessellation and triangulation analyses to distinguish breast cancers (76) as shown in Figure 6. Classification using multivariate techniques identified ratings for ductal against lobular carcinomas with up to $100 \%$ accuracy and up to $92 \%$ for benign to invasive cancers (76). Similar studies using the same quantification was further applied to lung and prostate tissue. In lung tissue, discrimination of normal from cancer lung tissue produced a $91 \%$ sensitivity and $92 \%$ specificity rate whereas, remarkably, small cell carcinomas were distinguished from non-small cell carcinomas with $100 \%$ sensitivity and specificity (77). Gao et al. also identified significant discrimination ( $P$ 0.01) of normal from cancerous prostate tissue. Together, these studies illustrate the consistently high classification rate of cancer types without the requirement of long tissue processing procedures. Further justification of its potential as a histological diagnostic tool would require high classification of the hundreds of cancer types, a role currently carried out by a histopathologist. 

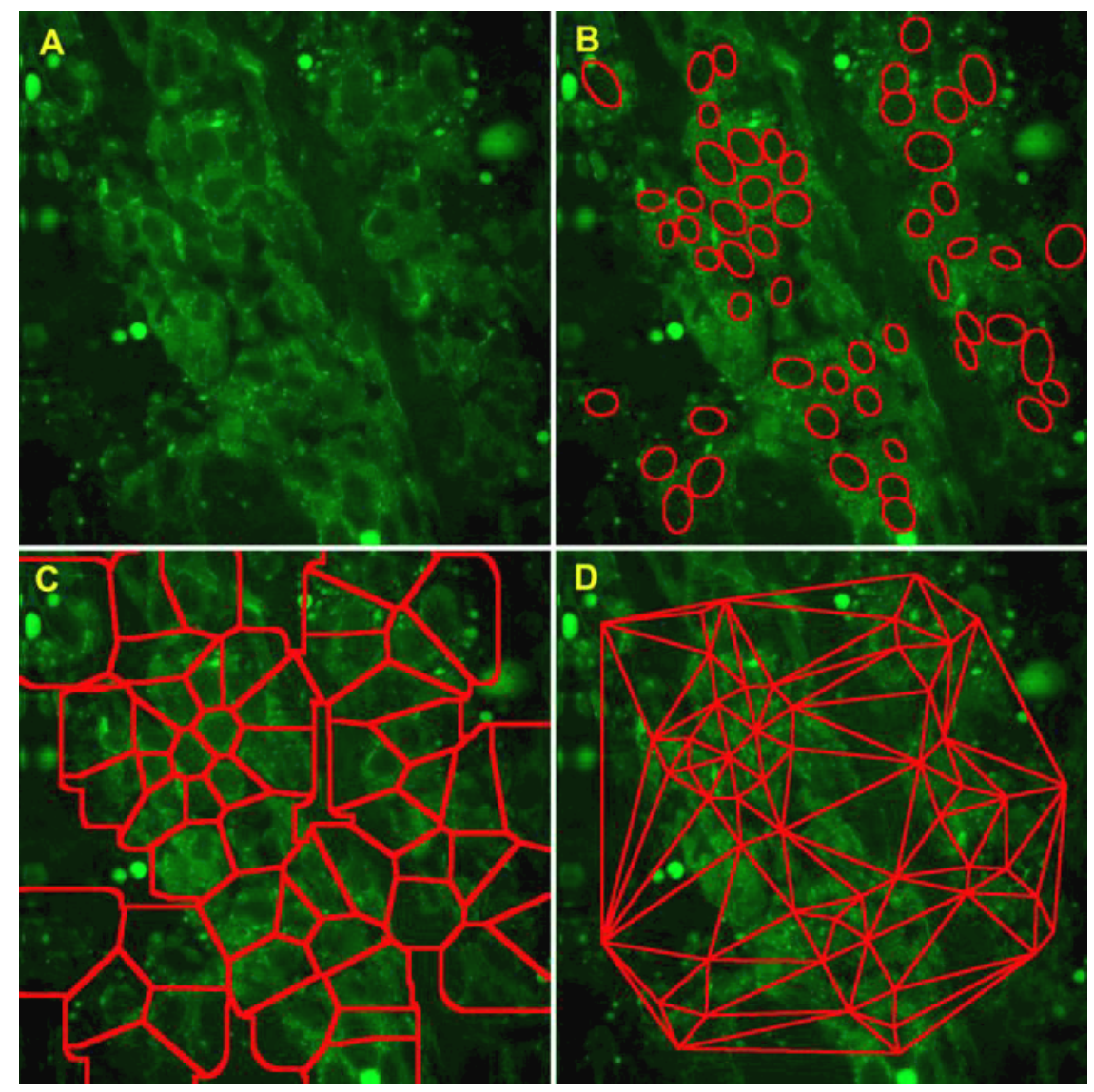

Figure 6. A CARS image (A) from the Z-stacks of an IDC sample, showing cell nuclei segmentation (B), Voronoi tessellation (C), and Delaunay triangulation (D). These analyses allowed differential diagnosis breast cancer sub-types. Image size: $120 \times 120 \mathrm{\mu m}^{2}$. Image from reference (76). Reproduced with permission.

As also described later in section 3.5, the role of CARS for imaging myelin in neurones has been well documented. In a study imaging the prostate, the presence of cavernous nerve bundle fibres could clearly be identified from its lipid-rich myelin (114). As radical prostatectomy for prostate cancer can sometimes cause erectile dysfunction due to damaging cavernous nerves, prognosis of surgery could be greatly improved with intra-operative applications and development of CARS laparoscopy. Meyer et al. carried out a study imaging lung metastases to the brain, as the biochemical information of the primary tumour differs remarkably to that of the brain; this allowed CARS tumour margin identification using the homogenous and delineated area of the primary tumour using CARS at 2845 $\mathrm{cm}^{-1}$. Additionally, individual tumour islets of homogenous dense tumour cells were also distinguishable in comparison to the heterogeneous brain tissue (115). With the advancement in technology for application of fibre optic CARS, it would be feasible that this high classification rate of cancers and delineation of tumour margins could also be applied surgically in-vivo, whilst also alleviating potential risks and side effects of surgery. 


\subsubsection{Insight into mechanisms of tumour invasion and metastases}

Understanding the mechanistic roles of cancer progression and invasion is essential for the development of rapid diagnostic tests and therapeutic treatments. It has been established that obesity and a high fat diet can enhance cancer metastases within the body as excess lipids can aggravate cancer $(116,117)$. Using the novel imaging capabilities of CARS and other NLO techniques, one can provide novel insights into the dynamics and mechanisms of cancer invasion.

A study using Balb/c cancer model mice fed on a high fat and lean diet were investigated for lipid effects in cancer development and progression (118). Using CARS imaging it was found that primary tumours in mouse models were unaffected by high lipid diet. However, two weeks after tumour implantation, high fat diet mice significantly induced circulating tumour cells. CARS and TPEF imaging in combination with intravital flow cytometry of mouse ear vein allowed identification of these circulating tumour cells containing high lipid content. The induction and promotion to metastatic cells could be attributed to free fatty acid incorporation in cancer cell membranes, reducing cell-cell contact and increasing surface adhesion $(118,119)$. Recently, as a clinical diagnostic technique metastatic circulating tumour cells in the blood were analysed for CARS signal intensity for intracellular lipids. A 7-fold increase in lipids was identified in metastatic cells compared to normal blood leukocytes providing a potential label-free biomarker for metastatic cancer (90). Mitra et al. additionally studied lipid mobilization of metastatic cells showing rapid lipid uptake and slow lipid mobilization kinetics in comparison to that of non-transformed RWPE-1 and non-malignant BPH-1 prostate epithelial cells. This high lipid affinity of metastatic cells could be manipulated to increase CARS sensitivity for clinical diagnosis (90).

Within tissue, the extracellular matrix plays an important role in homeostasis with many studies having identified its contribution to tumour progression and invasion (120). Current evidence indicates adipocytes have the role of an endocrine organ and potentially affect tumour progression (121). Epithelial-stromal interactions were imaged by CARS using mammary adipocytes of the fat pads or intraperitoneal adipose within a co-culture system for tumour cells. Unique findings of viable tumour cells present within epithelial-free fat pads concur with adipose as a maintenance factor of tumour cells whilst adipose tissue excised from different anatomical locations showed varied distinctive patterns of promoting tumour cell growth (122). From these studies, we can conclude that CARS has illustrated that the contents of the cellular microenvironment including lipids play a pivotal role in propagating invasion and metastases. Thus CARS can become an important tool for elucidating lipid related mechanisms in tumour cells. Moreover, development of a CARS flow 
cytometer could allow diagnosis of early stage metastatic cancers from a simple blood test (123, 124).

\subsection{Myelination of the nervous system}

The myelin sheath is a multilayer membrane, which wraps axons in the nervous system to provide electrical insulation and enable high-speed impulse propagation along the fibre. The myelin membranes contain about $70 \%$ lipid by weight and the high-density $\mathrm{CH}_{2}$ groups produce a large CARS signal $(125,126)$. The first report of CARS microscopy to image the myelin sheath was in explanted spinal cord tissues illustrating the node of Ranvier (127). Demyelination can cause loss of signal conduction across axons, characteristic in central nervous system (CNS) disorders such as multiple sclerosis or spinal cord injuries. CARS provides a non-invasive and label-free platform for imaging de-myelination of neurones during disease and re-myelination during repair.

Currently, CARS microscopy has been widely applied to label-free visualization of the myelin sheath in nerves from animal studies. From ex vivo tissue slices, mosaic images illustrating the presence of white matter in the cerebellum, fimbriae hippocampus and corpus collusum have been all captured in a high contrast single image $(89,115)$. As the causes of paranodal myelin damage in neuronal injuries are still obscure, studies to identify the mechanistic effect of chemical compounds such as lysophosphatidylcholine and glutamate were carried out in live spinal cord tissues $(88,128)$. Such studies using CARS can maintain high contrast without using invasive, non-specific labels which can photobleach. Both glutamate and lysophosphatidylcholine were shown to cause myelin splitting and demyelination respectively through subsequent calcium signalling and activation of calpain $(88,128$, 129). In a study carried out by Meyer et al. brain tumours were imaged using CARS at $2845 \mathrm{~cm}^{-1}$ for imaging lipids; areas of homogenous high lipid intensities identified delineated solid tumour regions which could be utilised for tumour margin identification in vivo (115). Unlike other microscopy and imaging techniques that require lengthy or fixed sample preparation, CARS imaging allows high resolution real-time imaging to shed light on the dynamic behaviour of myelin.

\subsection{Drug studies}

\subsubsection{Imaging drug effects on cellular metabolism}

Therapeutic drug interactions with cells initiate and alter biochemical signalling pathways leading to alterations of molecular moieties. An increased or decreased concentration of specific molecules in response to drug therapy in certain locations can produce CARS contrast which can be exploited by 
CARS imaging. Its capability of tracking alterations of cellular metabolism and molecular organisation makes CARS an asset as a qualitative and quantitative imaging platform to determine drug efficiency (82).

Recently, lipid droplet formation has been identified as an early marker of cell apoptosis (130). The induction of cellular apoptosis leads to an increase in mitochondrial membrane potential resulting in reactive oxygen species (ROS) formation. ROS inhibits the beta-oxidation of the fatty acyl-COA which normally leads to production of ATP. Instead, it is re-directed into lipid synthesis and lipid droplet formation. Thus the efficiency of chemotherapy drugs, which promote apoptosis, can be evaluated through the quantification of CARS active lipid droplet formation at various time points. We have demonstrated this cell based assay using three cancer drugs and show that campothecin and etoposide had similar chemotherapeutic effects while staurosporine a powerful drug induced higher levels of lipid droplet formation after 24 hours of incubation (93). This could potentially be through the activation of other mechanisms of apoptotic induction, warranting further investigation. In cardiovascular disease, statin therapy is the cornerstone treatment to alleviate symptoms by inhibiting the cholesterol biosynthesis pathway (91). Using multiplex CARS, various lipid types such as plate and needle shaped cholesterol crystals, lipid laden foam cells and extracellular lipids can be assessed in atherosclerosis. In ApoE -/- knockout mice, significant reduction of lipid accumulation after treatment with simvastatin was identified in intact atheromatous lesions, compared to that of non-treated mice (80). These studies indicate that multiplex CARS is an effective tool for pre-clinical drug screening and potentially in vivo assessment of chemotherapy treatments on tumours.

\subsubsection{Imaging for drug delivery and distribution}

Directly imaging unmodified drugs in cells requires a distinctive Raman peak signal stronger than the background from biochemicals in the cell. Due to the abundance of vibrational modes from different molecules in comparison to a single drug molecule, attaining such a peak could pose difficult especially in the Raman fingerprint region $(72,73)$. The spectral regions of $1800-2600 \mathrm{~cm}^{-1}$ are typically silent for most cellular biochemical constituents as only few specific moieties such as triple bonds, C-D vibrational modes have their peaks in this region and these are not normally present in cellular biomolecules. Therefore utilisation of molecules or Raman labels which have signals in this region can be exploited to track molecules using CARS. Deuterium commonly exploited by NMR spectroscopy has been used as a label in nanomedicines as the Raman peak of $\mathrm{CH}_{2}\left(2850 \mathrm{~cm}^{-1}\right)$ is shifted when deuterated to $\mathrm{CD}_{2}\left(2100 \mathrm{~cm}^{-1}\right)(131)$. A recent example is shown using deuterated Quaternary Ammonium Palmitoyl Glycol Chitosan (dGCPQ) particles of 30-50nm size which were tracked and pinpointed in mouse through the gastrointestinal tract, liver and gall bladder after oral 
administration as shown in Figure 7 (92). Use of isotopic labels in conjunction with CARS imaging provides a useful way to track and determine the pharmacodynamics of drugs without altering its properties.
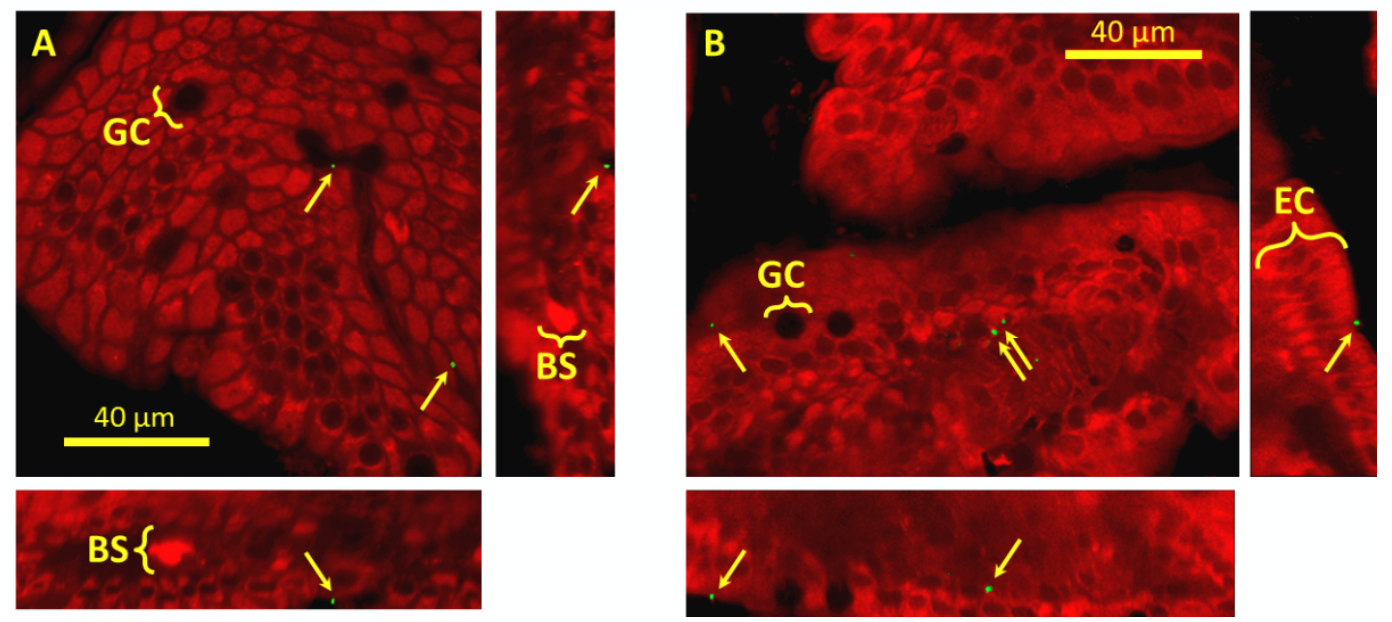

Figure 7. Orthogonal view reconstructions of depth-stack images of mouse ileum samples using contrast from TPF (red), SHG (blue) and epi-CARS tuned to $2100 \mathrm{~cm}^{-1}$ (green). This sample was harvested 120 minutes after oral dosing with dGCPQ6 and imaged from the external surface inwards. Particles (arrowed) are seen at the boundary between the muscularis propria and the Brunner's glands. Bile salts (BS) and erythrocytes (RBC) are marked for clarity. Image from reference (92). Copyright Wiley-VCH Verlag GmbH \& Co. KGaA. Reproduced with permission.

Drug sensitivity is a major contributor for the prognosis of a disease with many tumours acquiring resistance to certain chemotherapeutic drugs (132). Using label-free CARS set to identify the cell nucleus $\left(1096 \mathrm{~cm}^{-1}\right)$, TPEF was utilised to distinguish perinuclear distribution of inherent fluorescent doxorubicin around chemo-resistant MCF-7 cells (133). Targeting of individual drugs themselves using distinct vibrational peaks not present in high concentration within cells can alleviate the need for isotopes. The drug fluorouracil has distinct peaks for C-F vibrational modes (e.g. $637 \mathrm{~cm}^{-1}$ ) allowing one to identify its transformation in skin using Raman spectroscopy (134). A study to determine the distribution and drug release rate of paclitaxel from polymer compounds utilised CARS imaging directly for the unmodified drug. Through imaging at $3060 \mathrm{~cm}^{-1}$ for a paclitaxel vibrational mode, the in vivo biocompatibility of drug polymer based stents was determined to deliver drugs to the artery (135). Using peaks of increased intensity compared to that present in the surrounding tissue, its capability to produce contrast to identify the drug localisation has been demonstrated (136). These studies clearly indicate that label-free tracking of drugs is advancing dramatically and that many studies for their localisation and efficiency will increase with this advancement. 


\section{Scope and Challenges}

Among the available coherent Raman techniques, CARS is the most mature for translation to biomedical investigations and clinical studies. Its label-free, high resolution and chemical specific nature has allowed CARS to develop a niche for imaging lipids in biomedical studies with distinct advantages over conventional techniques such as mass spectrometry. Although non-resonant background observed in CARS imaging can lower signal to noise but efficient removal techniques are available and in certain cases this non-resonant background can be advantageous for viewing biological samples.

Furthermore CARS can be easily used as a powerful tool for application within histopathology departments for improved clinical evaluation. For example, CARS imaging is increasingly being utilised to uncover mechanistic and morphological information in plaque formation. Ultimately, a multimodal system with a compilation of other non-invasive techniques for various conditions and diseases such as atherosclerosis could provide highly detailed information for early stage diagnosis and formation of plaques $(137,138)$.

The ultimate challenge is the application of CARS during in vivo clinical procedures such as laparoscopy for on-site minimally invasive tissue diagnosis (139). The progression of this is hindered by challenges in the development of fibre based probes including those for endoscopy applications currently a prominent area of research in this field $(139,140)$. In general, successful application of CARS during such in vivo, clinical and surgical applications also critically rests on the development of robust, portable and low-cost lasers. Such a system requires the capability to deliver two ultrafast pulsed trains, effective signal collection of the backscattered CARS signal and adaption of a rapid scanning mechanism for an endoscopy approach $(139,140)$ along with a small foot-print fiber laser source $(56,57)$. Imaging techniques for motion reduction similar to that described for live animal myelin histomorphometry (141) will also assume importance for in vivo and clinical applications.

\section{Acknowledgements}

We acknowledge funding from EPSRC (EP/H028757/1 and EP/H028757/2), Royal Society Research Grant and Cambridge Cancer Centre (CCC) pump-priming award. We would like to thank Alexander Schreiner, Heather Ireland-Zechinni and Andy Watts for providing support and tissue samples for imaging. We would again also like to thank Ko et al., Garrett et al., Le et al., Thuc et al., Begin et al. and Okuno et al. for contributing images to this manuscript. 


\section{References}

1. C. K. Rosenthal, Nature Milestones in Light Microscopy Nature Cell Biol. 11, S6 (2009).

2. R. Hooke, Micrographia: or Some Physiological Descriptions of Minute Bodies, Made by Magnifying Glasses with Observations and Inquiries Thereupon. (James Allestry, 1665).

3. A. Van Leeuwenhoek, The Selected Works of Antony van Leeuwenhoek Containing His Microscopical Discoveries in Many of the Works of Nature

(Arno Press, 1977).

4. F. Zernike, Phase contrast, a new method for the microscopic observation of transparent objects. Physica 9, 974 (1942).

5. R. D. Allen, G. B. David, G. Nomarski, Zeiss-Nomarski differential interference equipment for transmitted-light microscopy. Z. Wiss. Mikrosk. 69, 193 (1969).

6. F. H. Smith, Microscopic interferometry. Research (London) 8, 385 (1955).

7. A. Heinrichs, Nature Milestones in Light Microscopy Nature Cell Biol. 11, S7 (2009).

8. O. Shimomura, F. H. Johnson, Y. Saiga, Extraction, purification and properties of aequorin, a bioluminescent protein from the luminous hydromedusan, Aequorea. Journal of cellular and comparative physiology 59, 223 (1962).

9. M. Chalfie, Y. Tu, G. Euskirchen, W. W. Ward, D. C. Prasher, Green fluorescent protein as a marker for gene expression. Science 263, 802 (1994).

10. R. Y. Tsien, The green fluorescent protein. Ann Rev Biochem 67, 509 (1998).

11. M. V. Matz et al., Fluorescent proteins from nonbioluminescent Anthozoa species. Nature biotechnology 17, 969 (1999).

12. R. Y. Tsien, Fluorescent probes of cell signaling. Annual review of neuroscience 12, 227 (1989).

13. A. Enejder, C. Brackmann, F. Svedberg, Coherent Anti-Stokes Raman Scattering Microscopy of Cellular Lipid Storage. IEEE J Quantum Elect 16, 506 (2010).

14. C. Krafft, B. Dietzek, M. Schmitt, J. Popp, Raman and coherent anti-Stokes Raman scattering microspectroscopy for biomedical applications. Journal of biomedical optics 17, 0408011 (2012).

15. R. L. McCreery, Raman Spectroscopy for Chemical Analysis. (Wiley-Interscience, 2005), vol. 225.

16. R. W. Minck, R. W. Terhune, W. G. Rado, Laser-Stimulated Raman Effect and Resonant FourPhoton Interactions in Gases. Appl Phys Lett 3, 181 (1963).

17. C. L. Evans, X. S. Xie, Coherent anti-stokes Raman scattering microscopy: chemical imaging for biology and medicine. Annu Rev Anal Chem 1, 883 (2008).

18. J. P. Pezacki et al., Chemical contrast for imaging living systems: molecular vibrations drive CARS microscopy. Nature Chemical Biology 7, 137 (Mar, 2011).

19. C. Krafft, B. Dietzek, M. Schmitt, J. Popp, Raman and coherent anti-Stokes Raman scattering microspectroscopy for biomedical applications. Journal of biomedical optics 17, 040801 (2012).

20. M. D. Duncan, J. Reintjes, T. Manuccia, Scanning coherent anti-Stokes Raman microscope. Opt Lett 7, 350 (1982).

21. A. Zumbusch, G. R. Holtom, X. S. Xie, Three-dimensional vibrational imaging by coherent anti-Stokes Raman scattering. Phys Rev Lett 82, 4142 (1999).

22. J. Cheng, A. Volkmer, L. Book, X. Xie, An epi-detected coherent anti-Stokes Raman scattering (E-CARS) microscope with high spectral resolution and high sensitivity. J Phys Chem B 105, 1277 (2001).

23. A. Volkmer, J. Cheng, Vibrational imaging with high sensitivity via epidetected coherent antiStokes Raman scattering microscopy. Phys Rev Lett 87023901, (2001).

24. J. Cheng, X. Xie, Coherent anti-Stokes Raman scattering microscopy: Instrumentation, theory, and applications. J Phys Chem B 108, 827 (2004). 
25. C. L. Evans et al., Chemical imaging of tissue in vivo with video-rate coherent anti-Stokes Raman scattering microscopy. P Natl Acad Sci USA 102, 16807 (2005).

26. B. G. Saar et al., Video-rate molecular imaging in vivo with stimulated Raman scattering. Science 330, 1368 (2010).

27. N. Liu et al., Label-Free Imaging Characteristics of Colonic Mucinous Adenocarcinoma Using Multiphoton Microscopy. Scanning, (2012).

28. X. Chen, O. Nadiarynkh, S. Plotnikov, P. J. Campagnola, Second harmonic generation microscopy for quantitative analysis of collagen fibrillar structure. Nat Protoc 7, 654 (2012).

29. B. Mallick, A. Lakshmanna, V. Radhalakshmi, S. Umapathy, Design and development of stimulated Raman spectroscopy apparatus using a femtosecond laser system. Current Science, 1551.

30. E. Ploetz, S. Laimgruber, S. Berner, W. Zinth, P. Gilch, Femtosecond stimulated Raman microscopy. Appl Phys B-Lasers O 87, 389 (2007).

31. C. Freudiger et al., Label-Free biomedical imaging with high sensitivity by stimulated Raman scattering microscopy. Science 322, 1857 (2008).

32. I. Rocha-Mendoza, W. Langbein, P. Borri, Coherent anti-Stokes Raman microspectroscopy using spectral focusing with glass dispersion. Appl Phys Lett 93, 201103 (2008).

33. H. Wang, Y. Fu, P. Zickmund, R. Shi, J.-X. Cheng, Coherent anti-stokes Raman scattering imaging of axonal myelin in live spinal tissues. Biophys J 89, 581 (2005).

34. E. O. Potma, D. J. Jones, J. X. Cheng, X. S. Xie, J. Ye, High-sensitivity coherent anti-Stokes Raman scattering microscopy with two tightly synchronized picosecond lasers. Opt Lett 27, 1168 (2002).

35. M. Müller, J. M. Schins, Imaging the Thermodynamic State of Lipid Membranes with Multiplex CARS Microscopy. The Journal of Physical Chemistry B 106, 3715 (2002/04/01, 2002).

36. M. Müller, A. Zumbusch, Coherent anti-Stokes Raman Scattering Microscopy. ChemPhysChem 8, 2156 (2007).

37. J.-x. Cheng, A. Volkmer, L. D. Book, X. S. Xie, Multiplex Coherent Anti-Stokes Raman Scattering Microspectroscopy and Study of Lipid Vesicles. The Journal of Physical Chemistry $B$ 106, 8493 (2002/08/01, 2002).

38. J. P. Day, G. Rago, K. F. Domke, K. P. Velikov, M. Bonn, Label-free imaging of lipophilic bioactive molecules during lipid digestion by multiplex coherent anti-Stokes Raman scattering microspectroscopy. Journal of the American Chemical Society 132, 8433 (2010).

39. J. Giordmaine, R. C. Miller, Tunable Coherent Parametric Oscillation in LiNbO3 at Optical Frequencies. Phys Rev Lett 14, 973 (1965).

40. W. Zhang, M. Parsons, G. McConnell, Flexible and stable optical parametric oscillator based laser system for coherent anti-Stokes Raman scattering microscopy. Microsc Res Techniq 73, 650 (2010).

41. F. Ganikhanov, C. L. Evans, B. G. Saar, X. S. Xie, High-sensitivity vibrational imaging with frequency modulation coherent anti-Stokes Raman scattering (FM CARS) microscopy. Opt Lett 31, 1872 (2006).

42. R. S. Lim et al., Multimodal CARS microscopy determination of the impact of diet on macrophage infiltration and lipid accumulation on plaque formation in ApoE-deficient mice. J Lipid Res 51, 1729 (2010).

43. P. F. Chimento et al., High-resolution narrowband CARS spectroscopy in the spectral fingerprint region. J Raman Spectrosc 40, 1229 (2009).

44. M. Okuno, H. Kano, P. Leproux, V. Couderc, H. O. Hamaguchi, Ultrabroadband multiplex CARS microspectroscopy and imaging using a subnanosecond supercontinuum light source in the deep near infrared. Opt Lett 33, 923 (May 1, 2008).

45. S. Begin et al., Coherent anti-Stokes Raman scattering hyperspectral tissue imaging with a wavelength-swept system. Biomedical optics express 2, 1296 (2011). 
46. W. Langbein, I. Rocha-mendoza, P. Borri, Single source coherent anti-Stokes Raman microspectroscopy using spectral focusing. Appl Phys Lett 95, 8 (2009).

47. I. Pope, W. Langbein, P. Watson, P. Borri, Simultaneous hyperspectral differential-CARS, TPF and SHG microscopy with a single $5 \mathrm{fs}$ Ti:Sa laser. Opt. Express 21, 7096 (03/25, 2013).

48. R. S. Lim et al., Identification of cholesterol crystals in plaques of atherosclerotic mice using hyperspectral CARS imaging. J Lipid Res 52, 2177 (2011).

49. B. von Vacano, L. Meyer, M. Motzkus, Rapid polymer blend imaging with quantitative broadband multiplex CARS microscopy. J Raman Spectrosc 38, 916 (2007).

50. C. Steuwe, C. F. Kaminski, J. J. Baumberg, S. Mahajan, Surface Enhanced Coherent AntiStokes Raman Scattering on Nanostructured Gold Surfaces. Nano Lett 11, 5339 (2011).

51. T. W. Kee, M. T. Cicerone, Simple approach to one-laser, broadband coherent anti-Stokes Raman scattering microscopy. Opt Lett 29, 2701 (2004).

52. V. Yakovlev, G. I. Petrov, Enhancing red-shifted white-light continuum generation in optical fibers for applications in nonlinear Raman microscopy. Opt Express 13, 1299 (2005).

53. R. Selm et al., Ultrabroadband background-free coherent anti-Stokes Raman scattering microscopy based on a compact Er:fiber laser system. Opt. Lett. 35, $3282(10 / 01,2010)$.

54. A. Gambetta et al., Fiber-format stimulated-Raman-scattering microscopy from a single laser oscillator. Opt Lett 35, 226 (2010).

55. A. F. Pegoraro et al., All-fiber CARS microscopy of live cells. Opt Express 17, 20700 (Nov 9, 2009).

56. M. Baumgartl et al., All-fiber laser source for CARS microscopy based on fiber optical parametric frequency conversion. Opt Express 20, 4484 (2012).

57. M. Baumgartl et al., Alignment-free, all-spliced fiber laser source for CARS microscopy based on four-wave-mixing. Opt Express 20, 21010 (2012).

58. J.-x. Cheng, A. Volkmer, L. D. Book, X. S. Xie, An Epi-Detected Coherent Anti-Stokes Raman Scattering (E-CARS) Microscope with High Spectral Resolution and High Sensitivity. J Phys Chem B 105, 1277 (2001).

59. A. Volkmer, J.-X. Cheng, X. Sunney Xie, Vibrational Imaging with High Sensitivity via Epidetected Coherent Anti-Stokes Raman Scattering Microscopy. Phys Rev Lett 87, 2 (2001).

60. J. Cheng, L. Book, Polarization coherent anti-Stokes Raman scattering microscopy. Opt Lett 26, 1341 (2001).

61. R. W. Hellwarth, Third-order optical susceptibilities of liquids and solids. Progress in Quantum Electronics 5, 1 (//, 1979).

62. M. Jurna, J. P. Korterik, C. Otto, J. L. Herek, H. L. Offerhaus, Background free CARS imaging by phase sensitive heterodyne CARS. Opt Express 16, 15863 (Sep 29, 2008).

63. G. Mueller et al., Determination and optimization of mode matching into optical cavities by heterodyne detection. Opt Lett 25, 266 (2000).

64. E. O. Potma, C. L. Evans, X. S. Xie, Heterodyne coherent anti-Stokes Raman scattering (CARS) imaging. Opt Lett 31, 241 (2006).

65. J. P. Ogilvie, E. Beaurepaire, A. Alexandrou, M. Joffre, Fourier-transform coherent anti-Stokes Raman scattering microscopy. Opt Lett 31, 480 (2006).

66. I. Rocha-Mendoza, W. Langbein, P. Watson, P. Borri, Differential coherent anti-Stokes Raman scattering microscopy with linearly chirped femtosecond laser pulses. Opt Lett 34, 2258 (2009).

67. J. Pawley, in Handbook Of Biological Confocal Microscopy, J. B. Pawley, Ed. (Springer US, 2006), pp. 20-42.

68. U. Neugebauer, J. H. Clement, T. Bocklitz, C. Krafft, J. Popp, Identification and differentiation of single cells from peripheral blood by Raman spectroscopic imaging. J Biophotonics 3, 579 (2010). 
69. Patel, Il et al., Segregation of human prostate tissues classified high-risk (UK) versus low-risk (India) for adenocarcinoma using Fourier-transform infrared or Raman microspectroscopy coupled with discriminant analysis. Anal Bioanal Chem 401, 969 (Aug, 2011).

70. A. Ghita, F. C. Pascut, M. Mather, V. Sottile, I. Notingher, Cytoplasmic RNA in Undifferentiated Neural Stem Cells: A Potential Label-Free Raman Spectral Marker for Assessing the Undifferentiated Status. Analytical chemistry 84, 3155 (2012).

71. N. Stone, C. Kendall, J. Smith, P. Crow, H. Barr, Raman spectroscopy for identification of epithelial cancers. Faraday discussions 126, 141 (2004).

72. C. Krafft, M. Kirsch, C. Beleites, G. Schackert, R. Salzer, Methodology for fiber-optic Raman mapping and FTIR imaging of metastases in mouse brains. Anal Bioanal Chem 389, 1133 (Oct, 2007).

73. Patel, II et al., High contrast images of uterine tissue derived using Raman microspectroscopy with the empty modelling approach of multivariate curve resolutionalternating least squares. The Analyst 136, 4950 (2011).

74. C. Petibois, Imaging methods for elemental, chemical, molecular, and morphological analyses of single cells. Anal Bioanal Chem 397, 2051 (Jul, 2010).

75. G. I. Petrov et al., Comparison of coherent and spontaneous Raman microspectroscopies for noninvasive detection of single bacterial endospores. Proc Natl Acad Sci U S A 104, 7776 (May 8, 2007).

76. Y. Yang et al., Differential diagnosis of breast cancer using quantitative, label-free and molecular vibrational imaging. Biomedical optics express 2, 2160 (2011).

77. L. Gao et al., Differential Diagnosis of Lung Carcinoma With Coherent Anti-Stokes Raman Scattering Imaging. Arch Pathol Lab Med 136, 1502 (Dec, 2012).

78. A. Downes, R. Mouras, A. Elfick, Optical Spectroscopy for Noninvasive Monitoring of Stem Cell Differentiation. J Biomed Biotech, (2010).

79. R. Mouras, P. O. Bagnaninchi, A. R. Downes, A. P. Elfick, Label-free assessment of adiposederived stem cell differentiation using coherent anti-Stokes Raman scattering and multiphoton microscopy. Journal of biomedical optics 17, 116011 (2012).

80. S. H. Kim et al., Multiplex coherent anti-stokes Raman spectroscopy images intact atheromatous lesions and concomitantly identifies distinct chemical profiles of atherosclerotic lipids. Circ Res 106, 1332 (2010).

81. C. Mörck et al., Statins inhibit protein lipidation and induce the unfolded protein response in the non-sterol producing nematode Caenorhabditis elegans. P Natl Acad Sci USA 106, 18285 (2009).

82. A. Pliss, A. N. Kuzmin, A. V. Kachynski, P. N. Prasad, Biophotonic probing of macromolecular transformations during apoptosis. P Natl Acad Sci USA 107, 12771 (2010).

83. J. Lin et al., Assessment of liver steatosis and fibrosis in rats using integrated coherent antiStokes Raman scattering and multiphoton imaging technique. Journal of biomedical optics 16, 116024 (2011).

84. J. P. Pezacki et al., Chemical contrast for imaging living systems: molecular vibrations drive CARS microscopy. Nature Chem Biol 7, 137 (2011).

85. T. T. Le, S. Yue, J. X. Cheng, Shedding new light on lipid biology with coherent anti-Stokes Raman scattering microscopy. J Lipid Res 51, 3091 (2010).

86. T. T. Le, A. Ziemba, Y. Urasaki, S. Brotman, G. Pizzorno, Label-free Evaluation of Hepatic Microvesicular Steatosis with Multimodal Coherent Anti-Stokes Raman Scattering Microscopy. PloS one 7, e51092 (2012).

87. C. Matthaus et al., In Vivo Characterization of Atherosclerotic Plaque Depositions by RamanProbe Spectroscopy and in Vitro Coherent Anti-Stokes Raman Scattering Microscopic Imaging on a Rabbit Model. Analytical chemistry 84, 7845 (2012). 
88. Y. Fu, H. Wang, T. B. Huff, R. Shi, J. X. Cheng, Coherent anti-Stokes Raman scattering imaging of myelin degradation reveals a calcium-dependent pathway in lyso-PtdCho-induced demyelination. Journal of neuroscience research 85, 2870 (2007).

89. Y. Fu, T. B. Huff, H. W. Wang, H. Wang, J. X. Cheng, Ex vivo and in vivo imaging of myelin fibers in mouse brain by coherent anti-Stokes Raman scattering microscopy. Opt Express 16, 19396 (2008).

90. R. Mitra, O. Chao, Y. Urasaki, O. B. Goodman, T. T. Le, Detection of Lipid-Rich Prostate Circulating Tumour Cells with Coherent Anti-Stokes Raman Scattering Microscopy. BMC Cancer 12, 540 (2012).

91. B. Sadowitz, K. G. Maier, V. Gahtan, Basic science review: Statin therapy--Part I: The pleiotropic effects of statins in cardiovascular disease. Vascular and endovascular surgery 44, 241 (2010).

92. N. L. Garrett, A. Lalatsa, I. Uchegbu, A. Schatzlein, J. Moger, Exploring uptake mechanisms of oral nanomedicines using multimodal nonlinear optical microscopy. J Biophoton 5, 458 (2012).

93. C. Steuwe et al., CARS based label-free assay for assessment of drugs by monitoring lipid droplets in tumour cells In Press, (2013).

94. E. Fuchs, V. Horsley, More than one way to skin. Genes Dev 22, 976 (2008).

95. T. Gambichler, R. Matip, G. Moussa, P. Altmeyer, K. Hoffmann, In vivo data of epidermal thickness evaluated by optical coherence tomography: effects of age, gender, skin type, and anatomic site. J Dermatological Sci 44, 145 (2006).

96. Patel, II et al., Isolating stem cells in the inter-follicular epidermis employing synchrotron radiation-based Fourier-transform infrared microspectroscopy and focal plane array imaging. Anal Bioanal Chem 404, 1745 (Oct, 2012).

97. K. Konig et al., Optical skin biopsies by clinical CARS and multiphoton fluorescence/SHG tomography. Laser Physics Lett 8, 465 (2011).

98. M. Zimmerley et al., Quantitative detection of chemical compounds in human hair with coherent anti-Stokes Raman scattering microscopy. Journal of biomedical optics 14, 044019 (2009).

99. H. G. Breunig et al., Combined in vivo multiphoton and CARS imaging of healthy and diseaseaffected human skin. Microsc Res Techniq 75, 492 (2012).

100. C. J. Strachan, M. Windbergs, H. L. Offerhaus, Pharmaceutical applications of non-linear imaging. Int J Pharmaceut 417, 163 (2011).

101. R. Ross, Atherosclerosis is an inflammatory disease. American heart journal 138, S419 (1999).

102. P. Duewell et al., NLRP3 inflammasomes are required for atherogenesis and activated by cholesterol crystals. Nature 464, 1357 (2010).

103. G. K. Hansson, Inflammation, atherosclerosis, and coronary artery disease. The New England journal of medicine 352, 1685 (2005).

104. G. Lucignani, M. Schafers, PET, CT and MRI characterisation of the atherosclerotic plaque. European journal of nuclear medicine and molecular imaging 37, 2398 (2010).

105. A. C. Ko et al., Multimodal nonlinear optical imaging of atherosclerotic plaque development in myocardial infarction-prone rabbits. Journal of biomedical optics 15, 020501 (Mar-Apr, 2010).

106. R. S. Lim et al., Multimodal CARS microscopy determination of the impact of diet on macrophage infiltration and lipid accumulation on plaque formation in ApoE-deficient mice. J Lipid Res 51, 1729 (2010).

107. W. C. Huang, G. B. Sala-Newby, A. Susana, J. L. Johnson, A. C. Newby, Classical macrophage activation up-regulates several matrix metalloproteinases through mitogen activated protein kinases and nuclear factor-kappaB. PloS one 7, e42507 (2012). 
108. L. P. Bechmann et al., The interaction of hepatic lipid and glucose metabolism in liver diseases. Journal of hepatology 56, 952 (2012).

109. J. Alexander, M. Torbenson, T. T. Wu, M. M. Yeh, Nonalcoholic Fatty Liver Disease Contributes to Hepatocellular Carcinoma in Non-cirrhotic Liver: A Clinical and Pathological Study. Journal of gastroenterology and hepatology, (2013).

110. D. E. Kleiner et al., Design and validation of a histological scoring system for nonalcoholic fatty liver disease. Hepatology 41, 1313 (2005).

111. M. A. G. Urena et al., Assessing risk of the use of livers with macro and microsteatosis in a liver transplant program. Transplant $P$ 30, 3288 (1998).

112. M. A. G. Urena et al., Hepatic steatosis in liver transplant donors: Common feature of donor population? World J Surg 22, 837 (1998).

113. M. Ramos et al., Ultrasound-guided excision combined with intraoperative assessment of gross macroscopic margins decreases the rate of reoperations for non-palpable invasive breast cancer. Breast, (2012).

114. L. Gao et al., Label-free high-resolution imaging of prostate glands and cavernous nerves using coherent anti-Stokes Raman scattering microscopy. Biomedical Opt Express 2, 915 (2011).

115. T. Meyer et al., Nonlinear microscopy, infrared, and Raman microspectroscopy for brain tumor analysis. Journal of biomedical optics 16, 021113 (2011).

116. N. Alikhani et al., Mammary tumor growth and pulmonary metastasis are enhanced in a hyperlipidemic mouse model. Oncogene, (2012).

117. L. Yan, L. C. DeMars, Effects of dietary fat on spontaneous metastasis of Lewis lung carcinoma in mice. Clinical \& experimental metastasis 27, 581 (2010).

118. T. T. Le, T. B. Huff, J. X. Cheng, Coherent anti-Stokes Raman scattering imaging of lipids in cancer metastasis. BMC Cancer 9, 42 (2009).

119. U. N. Das, Essential fatty acids and their metabolites as modulators of stem cell biology with reference to inflammation, cancer, and metastasis. Cancer metastasis reviews 30, 311 (2011).

120. J. Park, D. M. Euhus, P. E. Scherer, Paracrine and endocrine effects of adipose tissue on cancer development and progression. Endocrine reviews 32, 550 (2011).

121. J. Park, P. E. Scherer, Adipocyte-derived endotrophin promotes malignant tumor progression. The Journal of clinical investigation 122, 4243 (2012).

122. T. S. Salameh et al., An ex vivo co-culture model system to evaluate stromal-epithelial interactions in breast cancer. International journal of cancer. Journal international du cancer 132, 288 (2013).

123. C. H. Camp, Jr., S. Yegnanarayanan, A. A. Eftekhar, A. Adibi, Label-free flow cytometry using multiplex coherent anti-Stokes Raman scattering (MCARS) for the analysis of biological specimens. Opt Lett 36, 2309 (2011).

124. C. H. Camp, Jr., S. Yegnanarayanan, A. A. Eftekhar, H. Sridhar, A. Adibi, Multiplex coherent anti-Stokes Raman scattering (MCARS) for chemically sensitive, label-free flow cytometry. Opt Express 17, 22879 (2009).

125. J. P. Doyle, D. R. Colman, Glial-neuron interactions and the regulation of myelin formation. Current opinion in cell biology 5, 779 (1993).

126. W. T. Norton, S. E. Poduslo, Myelination in rat brain: changes in myelin composition during brain maturation. Journal of neurochemistry 21, 759 (1973).

127. H. Wang, Y. Fu, P. Zickmund, R. Shi, J. X. Cheng, Coherent anti-stokes Raman scattering imaging of axonal myelin in live spinal tissues. Biophys J 89, 581 (2005).

128. Y. Fu, W. Sun, Y. Shi, R. Shi, J. X. Cheng, Glutamate excitotoxicity inflicts paranodal myelin splitting and retraction. PloS one 4, e6705 (2009).

129. T. B. Huff et al., Real-time CARS imaging reveals a calpain-dependent pathway for paranodal myelin retraction during high-frequency stimulation. PloS one 6, e17176 (2011). 
130. J. Boren, K. M. Brindle, Apoptosis-induced mitochondrial dysfunction causes cytoplasmic lipid droplet formation. Cell death and differentiation 19, 1561 (2012).

131. A. E. Mutlib, Application of stable isotope-labeled compounds in metabolism and in metabolism-mediated toxicity studies. Chemical research in toxicology 21, 1672 (2008).

132. L. Porcelli et al., Synthetic lethality to overcome cancer drug resistance. Current medicinal chemistry 19, 3858 (2012).

133. R. Mouras, G. Rischitor, A. Downes, D. Salter, A. Elfick, Nonlinear optical microscopy for drug delivery monitoring and cancer tissue imaging. J Raman Spectrosc 41, 848 (2010).

134. G. Zhang, D. J. Moore, K. B. Sloan, C. R. Flach, R. Mendelsohn, Imaging the prodrug-to-drug transformation of a 5-fluorouracil derivative in skin by confocal Raman microscopy. The Journal of investigative dermatology 127, 1205 (2007).

135. E. Kang et al., In situ visualization of paclitaxel distribution and release by coherent antiStokes Raman scattering microscopy. Analytical chemistry 78, 8036 (2006).

136. I. Latka et al., Spectroscopic detection of chemotherapeutics and antioxidants. Proc Spie 8427, (2012).

137. S. C. van den Oord et al., Assessment of subclinical atherosclerosis using contrast-enhanced ultrasound. European heart journal cardiovascular Imaging 14, 56 (2013).

138. M. R. Jones et al., Intravascular frequency-domain optical coherence tomography assessment of atherosclerosis and stent-vessel interactions in human carotid arteries. AJNR. American journal of neuroradiology 33, 1494 (2012).

139. M. Balu, G. Liu, Z. Chen, B. J. Tromberg, E. O. Potma, Fiber delivered probe for efficient CARS imaging of tissues. Opt Express 18, 2380 (2010).

140. L. Fu, M. Gu, Fibre-optic nonlinear optical microscopy and endoscopy. J Microsc 226, 195 (Jun, 2007).

141. E. Belanger et al., Live animal myelin histomorphometry of the spinal cord with video-rate multimodal nonlinear microendoscopy. Journal of biomedical optics 17, 021107 (2012). 